\title{
Amino acid needs of lactating dairy cows: Predicting limiting amino acids in contemporary rations fed to high producing dairy cattle in California using metabolic models
}

\author{
N. Swanepoel ${ }^{\mathrm{a}, *}$, P.H. Robinson ${ }^{\mathrm{b}}$, L.J. Erasmus ${ }^{\mathrm{a}}$ \\ a Department of Animal and Wildife Sciences, University of Pretoria, Pretoria 0001, South Africa \\ b Department of Animal Science, University of California, Davis, CA 95616, USA
}

\section{A R T I C L E I N F O}

\section{Article history:}

Received 16 February 2010

Received in revised form 2 August 2010

Accepted 6 August 2010

Available online $\mathrm{xxx}$

\section{Keywords:}

Amino acid profile

Corn crude protein

Metabolic models

Ruminally protected AA

\begin{abstract}
A B S T R A C T
The objectives were to predict amino acid (AA) profiles of intestinally delivered protein in California high group (i.e., lactating but not yet confirmed to be in calf) dairy cattle fed contemporary rations using three metabolic models of dairy cows. This was done in order to predict limiting AA in dairy rations to determine if there was enough consistency in the nutrient profiles of these rations to support a common ruminally protected (RP) AA package to supplement similar rations. Nutrient profiles of 16 commercial high group dairy cow rations were evaluated, and limiting AA predicted by the metabolic models 'Amino Cow', 'CPM Dairy' and 'Shield'. Higher inclusion levels of corn products in rations increased the contribution of corn CP to the total CP content of the total mixed ration (TMR), from 0.20 to 0.40 . Even though the lysine to methionine ratio decreased as more corn $\mathrm{CP}$ was included in the TMR, it did not have a major impact on the final predicted AA profile of metabolizable protein (MP), but MP delivery (g/d) to the duodenum was predicted to decrease with increased corn $\mathrm{CP}$ levels. None of the models predicted any affect of increased corn CP levels on milk components but, according to Shield, it did have an effect on milk yield which increased when the ratio of lysine to methionine in MP decreased. The sequence of AA limitation among rations was the same within model, but differed substantially among models. Methionine, isoleucine and lysine were predicted to be most limiting according to Amino Cow, CPM Dairy and Shield, respectively. There appears to be sufficient consistency in nutrient profiles among rations to support a ruminally protected AA complex to balance the model predicted AA profile in order to increase animal productivity and efficiency of utilization of nutrients. There is no absolute way to decide which model predictions are most correct. However because Shield predictions suggested a higher correlation between Lys and Met in MP and production, as well as predicted AA ratios to milk responses related to these ratios, use of the Shield predicted AA package is supported.
\end{abstract}

(C) 2010 Published by Elsevier B.V.

Abbreviations: AA, amino acids; ADF, acid detergent fiber; ADICP, acid detergent insoluble CP; CP, crude protein; DDG, dried distillers grains; DIM, days in milk; $\mathrm{dNDF}_{30}, 30 \mathrm{~h}$ ruminal in vitro aNDF digestibility; DHIA, Dairy Herd Improvement Association; DM, dry matter; EAA, essential AA; EE, ether extract; MCP, microbial CP; MP, metabolizable protein; NDF, neutral detergent fiber; aNDF, amylase-treated NDF; aNDFom, aNDF expressed free of residual ash; $\mathrm{RP}$, rumen protected; SolCP, soluble CP; TMR, total mixed ration.

* Corresponding author at: Meadow Feeds, Warich Close Office Park, 39 Van Vuuren Street, Roodepoort 1737, South Africa. Tel.: +27 119916000.

E-mail address: nanswanepoel@gmail.com (N. Swanepoel). 


\section{Introduction}

Over the past 10 years there has been a substantial increase in the number of motor vehicle fuel ethanol distillation plants in the Midwestern USA, primarily using corn grain as their feedstock, creating vast quantities of corn distillers' by-products. Dairy rations in many parts of the USA have long depended upon corn based feedstuffs (e.g., corn grain, corn silage, corn gluten, as well as germ feeds and meals) and, with the widespread increase in use of corn dried distillers grains (DDG), it is not uncommon to find $300-400 \mathrm{~g} / \mathrm{kg}$ of total crude protein (CP) in total mixed rations (TMR) originating from corn products.

Corn proteins have long been recognized to have an amino acid (AA) profile that is poorly matched to that of milk protein produced by dairy cows (Schwab et al., 1976; NRC, 2001), primarily due to its low lysine content. Belyea et al. (1989) demonstrated the high variability in nutrient content that is inherent to by-product feeds, mostly due to differences in processing methods among plants and changes in these methods over time. Increased CP levels in rations, as a result of increased inclusion of less expensive protein sources to keep ration costs low, or as a safety factor due to uncertainty of feed composition to ensure that animal requirements for limiting AA are met (St-Pierre and Thraen, 1999), may lead to increased excretion of $\mathrm{N}$ in urine and feces. This is in direct opposition to recent efforts designed to minimize negative impacts of dairy cows on the environment.

Researchers and nutritionists differ on which AA are limiting for milk production in dairy cattle, but studies have suggested lysine and methionine to be the most likely candidates (Burris et al., 1976; Schwab et al., 1976, 1982) followed by phenylalanine, isoleucine, threonine (Derrig et al., 1974; Vik-Mo et al., 1974; Nichols et al., 1998; Piepenbrink and Schingoethe, 1998; Liu et al., 2000) histidine and arginine (Vanhatalo et al., 1999). Balancing diets for MP is difficult using current metabolic models due to a lack of accurate predictability of intestinally absorbable AA needs and delivery. Models cannot fully account for variability among raw materials, cows, environment or their interaction, which limits the application of their predictions. However, more information is required regarding limiting AA, and the effect of supplementing them, and since large scale dose-response studies are very difficult to conduct with lactating cows, comparing model predicted limiting AA in various, well-defined, rations could help to understand and estimate nutrient supplies to the cows and make ration formulation based on AA levels in intestinally absorbed protein feasible. Since results from previous studies in which only lysine was supplemented were inconsistent (Robinson, 2010), and results from a study we conducted showed a substantial negative response (Swanepoel et al., 2010), it raises questions as to whether lysine is limiting in contemporary California dairy rations.

This study was an evaluation of feeding practices, including sampling of feeds and TMR on selected California herds, which were then evaluated with three metabolic models used by nutritionists, to determine model predicted AA profiles of intestinally delivered protein in order to identify limiting AA and to determine if there is enough consistency in the nutrient profiles of these rations to justify production of a ruminally protected (RP) AA complex which could provide cows with an 'ideal' dietary AA profile to improve animal production and efficiency.

\section{Materials and methods}

\subsection{Farm, cows and management}

A group of 24 potential dairy farm co-operators were identified in Tulare and Kings Counties (CA, USA), the two main milk producing counties of California. Dairies chosen for this initial list were judged to be representative of dairy farms in the respective counties and milked more than 1000 cows. From the total of 24 dairies, 16 were finally chosen based on an assessment of factors including ration composition, standard/level of management on the dairy (i.e., accurate mixing and feeding records to determine amounts of feed mixed and TMR fed), use of computerized herd record and management systems and an organized structural outlay of the lactation pens. Each dairy had a consulting nutritionist responsible for formulating the ration, and care was taken during the selection process to select dairies with different nutritionists.

\subsection{Sample collection}

Three visits to each farm were scheduled to coincide with the regular Dairy Herd Improvement Association (DHIA) milk test. During the first visit, dairies were appraised and the managers informed of the procedures to follow. One of the high production pens was identified for use in the survey at each farm.

During the second visit, TMR preparation was observed before TMR samples were collected from the bunks as they was being fed to the specified pen. Six handfuls of TMR were collected at evenly spaced locations along the bunk-line, pooled and the entire sample quartered, keeping two opposite quarters for analysis. When TMR samples contained whole citrus pulp, large pieces were broken up by hand before quartering to ensure accurate sampling.

Commodity feeds and silages, mixed into the TMR, were identified and sampled by taking four to five handfuls of each. A 'golf club' hay probe (Seifert Analytical, Lodi, CA, USA) was used to take 12-16 core samples from all hays as well as oat, wheat and rice straws. 
A second TMR sample was collected, after preparation was again observed prior to, or on the day of, regular DHIA milk testing following the same procedures as above. Highly variable wet commodities, such as green alfalfa chop, were also sampled a second time. As far as possible, the two sampling visits were scheduled at different feeding times during the day.

All feed and TMR samples were stored in a refrigerator and later transferred to a freezer $\left(-19^{\circ} \mathrm{C}\right)$ until drying for chemical analysis. Chemical compositions obtained from previous studies were used for ingredients that were difficult to sample, such as liquid whey, molasses and corn syrup, as well as feed additives with standard or constant chemical compositions, such as yeast cultures, ruminally inert fats and rumen buffers.

Information on farm, cow and pen characteristics, mixing equipment, feeding sequences and any anomalies were recorded for each dairy. The amount of TMR refused, and frequency of removal, was also recorded. A herd records file with milk production and composition data from the most recent DHIA milk test (i.e., milk yield, true protein and fat proportions, somatic cell counts (SCC), days in milk (DIM) and lactation numbers), was downloaded prior to the start of the project, and again after the DHIA milk test results were entered.

Depending on the method used to monitor mixing and feeding, feed delivery records were collected for at least 5 days prior to the milk test from computerized programs or TMR mix sheets. Mixing information was used to calculate dry matter (DM) intake/pen.

\subsection{Analytical methods}

\subsubsection{Feed preparation and assays}

All TMR samples, silages and other wet ingredients were weighed before being dried at $55^{\circ} \mathrm{C}$ for $48 \mathrm{~h}$. All samples were removed and left to equilibrate for $24 \mathrm{~h}$ before they were bagged, weighed and tagged for analysis.

All samples were ground to pass a $1 \mathrm{~mm}$ screen using a model 4 Wiley Mill (Thomas Scientific, Swedesboro, NJ, USA). Feed and TMR samples were analyzed for DM, ash, neutral detergent fiber (aNDFom), acid detergent fiber (ADFom), lignin treated with sulphuric acid (lignin(sa)), starch, free sugars (soluble carbohydrates), CP, acid detergent insoluble CP (ADICP), minerals (TMR samples only), fat (EE), $30 \mathrm{~h}$ ruminal in vitro aNDF digestibility ( $\mathrm{dNDF}_{30}$ ) and soluble $\mathrm{CP}(\mathrm{SolCP})$ as described by Swanepoel et al. (2010).

\subsubsection{Model evaluation}

Once all cow and feed assay information was collected and tabulated, the nutrient profiles of the 16 rations were evaluated using the metabolic models Amino Cow (2007), CPM Dairy (2006) and Shield (Robinson, 2009). These models are all largely empirical, but with different AA levels assigned to feeds and microbial CP (MCP).

In all cases, cow information, calculated ingredient composition of the TMR and chemical composition of the feeds that were fed was entered into the models as required by model. All default feed components were used with the exception of feed DM, CP, ADFom, aNDFom and fat for Amino Cow, DM, CP, SolCP, ADICP, ADFom, aNDFom, lignin(sa), ash, fat, sugars and starch for CPM Dairy, and DM, OM, fat, CP, SolCP, ADICP, aNDFom and $\mathrm{dNDF}_{30}$ for Shield.

\section{Results}

Results were divided into those that were measured and those that were model predicted. All feed and TMR samples collected and analyzed, as well as feeding and animal production data collected from the farms are measured values. Values and correlations drawn from models were predicted using the information gathered on the farms and thus are defined as predicted. The ration and model evaluation process determined relationships between variables to assess possible correlations among variables, even though this does not imply cause and effect.

\subsection{Measured results}

\subsubsection{Ration evaluation}

Where numerous samples of the same ingredient were collected, a subset of samples was pooled to obtain an average with a standard error (SE), except for corn DDG where all samples were assayed (Table 1). These average values were used in model evaluation. The composition of the ingredients was consistent among dairies, with only minor differences in a few nutrients and wet ingredients such as citrus pulp.

Forages were collected and assayed separately by dairy due to higher variation among them. Averages and SE of forages are in Table 2, but individual farm values were used in model evaluations. Alfalfa fresh chop was sampled at both farm visits, since it was cut daily leading to compositional differences among days. Alfalfa hay was divided into high or low quality (as defined by the dairy) when two sources were sampled, but there was little chemical difference between them. Forage composition was relatively consistent among dairies, with the possible exception of whole crop wheat silage.

Chemical composition of the two TMR samples from each dairy was analyzed separately and averaged (Table 3). The values for the 16 dairies, and minimum NRC (2001) recommendations where appropriate, are listed for comparison. Almost all major nutrient requirements were met by the $16 \mathrm{TMR}$, with no substantive nutrient undersupply on any dairy. There 
Table 1

Chemical analysis (+standard deviation if enough samples were collected) of commodity ingredients $\left(\mathrm{g} / 100 \mathrm{~g} \mathrm{DM} \mathrm{DM}^{\mathrm{a}}\right)$ used in total mixed rations of the 16 dairies.

\begin{tabular}{|c|c|c|c|c|c|c|c|c|c|c|c|c|c|}
\hline & $n$ & $\mathrm{DM}^{\mathrm{a}}$ & $\mathrm{OM}^{\mathrm{b}}$ & $\mathrm{CP}^{\mathrm{c}}$ & $\mathrm{ADICP}^{\mathrm{d}}$ & SolCPe & $\mathrm{aNDF}_{\mathrm{om}^{\mathrm{g}}}{ }^{\mathrm{g}}$ & $\mathrm{dNDF}_{30}{ }^{\mathrm{f}}$ & $\mathrm{ADF}_{\mathrm{om}}{ }^{\mathrm{h}}$ & $\operatorname{Lignin}(\mathrm{sa})^{\mathrm{i}}$ & Starch & Fat & Sugars \\
\hline Almond hulls & $5^{1}$ & $\begin{array}{l}93.00 \\
(1.344)^{\mathrm{j}}\end{array}$ & $\begin{array}{l}92.20 \\
(0.453)\end{array}$ & $\begin{array}{l}5.69 \\
(0.172)\end{array}$ & $\begin{array}{l}25.66 \\
(2.040)\end{array}$ & $\begin{array}{l}40.36 \\
(1.550)\end{array}$ & $\begin{array}{l}34.72 \\
(1.706)\end{array}$ & $\begin{array}{l}31.67 \\
(3.730)\end{array}$ & $\begin{array}{l}28.50 \\
(1.113)\end{array}$ & $\begin{array}{l}10.99 \\
(0.502)\end{array}$ & $\begin{array}{l}1.72 \\
(0.351)\end{array}$ & $\begin{array}{l}2.45 \\
(0.268)\end{array}$ & $\begin{array}{l}17.81 \\
(0.591)\end{array}$ \\
\hline Barley, rolled & 1 & 91.00 & 97.19 & 12.19 & 2.56 & 22.29 & 21.50 & 55.70 & 7.80 & 2.00 & 50.70 & 1.74 & 1.30 \\
\hline Beet pulp shreds & 2 & 94.10 & 93.93 & 9.52 & 3.94 & 42.97 & 32.25 & 86.36 & 20.15 & 0.83 & 6.43 & 0.74 & 18.80 \\
\hline Brandy pomace & 1 & 30.94 & 89.96 & 10.75 & 37.79 & 30.80 & 39.70 & 31.16 & 43.90 & 21.70 & 0.50 & 2.50 & 0.20 \\
\hline Canola pellets & $4^{1}$ & $\begin{array}{l}91.15 \\
(0.380)\end{array}$ & $\begin{array}{l}91.59 \\
(0.197)\end{array}$ & $\begin{array}{l}42.72 \\
(0.324)\end{array}$ & $\begin{array}{l}6.09 \\
(1.560)\end{array}$ & $\begin{array}{l}33.40 \\
(0.510)\end{array}$ & $\begin{array}{l}24.38 \\
(1.186)\end{array}$ & $\begin{array}{l}45.40 \\
(2.000)\end{array}$ & $\begin{array}{l}18.55 \\
(1.154)\end{array}$ & $\begin{array}{l}7.63 \\
(1.014)\end{array}$ & $\begin{array}{l}2.83 \\
(0.782)\end{array}$ & $\begin{array}{l}3.95 \\
(0.108)\end{array}$ & $\begin{array}{l}6.53 \\
(0.312)\end{array}$ \\
\hline Carrot pulp & 1 & 12.47 & 93.51 & 7.31 & 8.12 & 54.33 & 26.45 & 85.20 & 24.70 & 1.25 & 3.10 & 1.41 & 1.65 \\
\hline Citrus pulp & 3 & $\begin{array}{l}26.31 \\
(4.312)\end{array}$ & $\begin{array}{l}91.72 \\
(0.506)\end{array}$ & $\begin{array}{l}9.30 \\
(1.200)\end{array}$ & $\begin{array}{l}6.47 \\
(1.275)\end{array}$ & $\begin{array}{l}56.91 \\
(4.200)\end{array}$ & $\begin{array}{l}19.08 \\
(2.010)\end{array}$ & $\begin{array}{l}77.81 \\
(3.810)\end{array}$ & $\begin{array}{l}25.30 \\
(4.790)\end{array}$ & $\begin{array}{l}0.82 \\
(0.017)\end{array}$ & $\begin{array}{l}1.13 \\
(0.277)\end{array}$ & $\begin{array}{l}1.76 \\
(0.394)\end{array}$ & $\begin{array}{l}3.05 \\
(1.449)\end{array}$ \\
\hline Corn gluten feed & 2 & 91.80 & 91.91 & 23.53 & 1.85 & 51.60 & 30.55 & 61.80 & 9.55 & 1.00 & 14.50 & 3.37 & 1.00 \\
\hline Corn grain, flaked & $3^{1}$ & $\begin{array}{l}85.53 \\
(0.384)\end{array}$ & $\begin{array}{l}98.75 \\
(0.062)\end{array}$ & $\begin{array}{l}8.68 \\
(0.554)\end{array}$ & $\begin{array}{l}0.00 \\
-\end{array}$ & $\begin{array}{l}25.50 \\
(3.080)\end{array}$ & $\begin{array}{l}8.27 \\
(0.233)\end{array}$ & $\begin{array}{l}66.60 \\
(2.690)\end{array}$ & $\begin{array}{l}3.10 \\
(0.153)\end{array}$ & $\begin{array}{l}0.40 \\
(0.100)\end{array}$ & $\begin{array}{l}73.07 \\
(2.335)\end{array}$ & $\begin{array}{l}2.59 \\
(0.704)\end{array}$ & $\begin{array}{l}0.50 \\
(0.153)\end{array}$ \\
\hline Cottonseed, whole linted & $3^{1}$ & $\begin{array}{l}93.17 \\
(1.040)\end{array}$ & $\begin{array}{l}95.69 \\
(0.047)\end{array}$ & $\begin{array}{l}21.34 \\
(0.740)\end{array}$ & $\begin{array}{l}7.39 \\
(0.997)\end{array}$ & $\begin{array}{l}23.00 \\
(0.010)\end{array}$ & $\begin{array}{l}43.17 \\
(2.282)\end{array}$ & $\begin{array}{l}9.30 \\
(0.750)\end{array}$ & $\begin{array}{l}33.83 \\
(1.633)\end{array}$ & $\begin{array}{l}9.80 \\
(0.600)\end{array}$ & $\begin{array}{l}0.50 \\
-\end{array}$ & $\begin{array}{l}20.18 \\
(1.196)\end{array}$ & $\begin{array}{l}0.67 \\
-\end{array}$ \\
\hline Cottonseed, ground pima & 3 & $\begin{array}{l}93.30 \\
(0.252)\end{array}$ & $\begin{array}{l}95.17 \\
(0.076)\end{array}$ & $\begin{array}{l}23.34 \\
(1.164)\end{array}$ & $\begin{array}{l}6.76 \\
(0.649)\end{array}$ & $\begin{array}{l}25.30 \\
(2.560)\end{array}$ & $\begin{array}{l}36.92 \\
(2.695)\end{array}$ & $\begin{array}{l}31.40 \\
(8.990)\end{array}$ & $\begin{array}{l}28.40 \\
(1.595)\end{array}$ & $\begin{array}{l}10.22 \\
(0.505)\end{array}$ & $\begin{array}{l}0.53 \\
(0.033)\end{array}$ & $\begin{array}{l}22.49 \\
(0.531)\end{array}$ & $\begin{array}{l}0.52 \\
(0.060)\end{array}$ \\
\hline Distillers grains, dried & $6^{1}$ & $\begin{array}{l}91.93 \\
(0.400)\end{array}$ & $\begin{array}{l}95.61 \\
(0.025)\end{array}$ & $\begin{array}{l}30.84 \\
(0.550)\end{array}$ & $\begin{array}{l}7.51 \\
(1.804)\end{array}$ & $\begin{array}{l}25.29 \\
(2.400)\end{array}$ & $\begin{array}{l}31.12 \\
(1.096)\end{array}$ & $\begin{array}{l}53.20 \\
(2.900)\end{array}$ & $\begin{array}{l}11.65 \\
(0.792)\end{array}$ & $\begin{array}{l}1.83 \\
(0.475)\end{array}$ & $\begin{array}{l}4.55 \\
(1.136)\end{array}$ & $\begin{array}{l}11.99 \\
(0.583)\end{array}$ & $\begin{array}{l}0.58 \\
(0.149)\end{array}$ \\
\hline Distillers grains, wet & 3 & $\begin{array}{l}32.99 \\
(0.708)\end{array}$ & $\begin{array}{l}96.78 \\
(0.162)\end{array}$ & $\begin{array}{l}36.03 \\
(0.883)\end{array}$ & $\begin{array}{l}14.10 \\
(1.132)\end{array}$ & $\begin{array}{l}29.55 \\
(3.370)\end{array}$ & $\begin{array}{l}30.80 \\
(0.777)\end{array}$ & $\begin{array}{l}54.10 \\
(2.930)\end{array}$ & $\begin{array}{l}16.83 \\
(0.977)\end{array}$ & $\begin{array}{l}2.47 \\
(0.203)\end{array}$ & $\begin{array}{l}3.03 \\
(0.318)\end{array}$ & $\begin{array}{l}10.37 \\
(0.183)\end{array}$ & $\begin{array}{l}0.20 \\
N D^{k}\end{array}$ \\
\hline Linseed meal & 1 & 91.80 & 92.03 & 43.70 & 2.79 & 28.23 & ND & 68.49 & 14.60 & 5.75 & 2.55 & 2.04 & 2.45 \\
\hline Linseed pellets & 1 & 91.90 & 92.39 & 35.19 & 3.73 & 25.76 & 32.60 & 36.56 & 24.40 & 7.10 & 2.60 & 2.22 & 4.50 \\
\hline Raisin tailings & 1 & 92.20 & 90.28 & 8.20 & 24.01 & 41.89 & 21.10 & 40.72 & 24.90 & 10.65 & 0.50 & 0.39 & 26.20 \\
\hline Soybean meal & 3 & $\begin{array}{l}91.23 \\
(0.260)\end{array}$ & $\begin{array}{l}92.46 \\
(0.128)\end{array}$ & $\begin{array}{l}51.10 \\
(0.749)\end{array}$ & $\begin{array}{l}0.32 \\
(0.317)\end{array}$ & $\begin{array}{l}21.25 \\
(0.050)\end{array}$ & $\begin{array}{l}8.50 \\
(0.702)\end{array}$ & $\begin{array}{l}69.60 \\
(0.240)\end{array}$ & $\begin{array}{l}5.10 \\
(0.379)\end{array}$ & $\begin{array}{l}0.20 \\
(0.058)\end{array}$ & $\begin{array}{l}5.17 \\
(0.491)\end{array}$ & $\begin{array}{l}0.58 \\
(0.106)\end{array}$ & $\begin{array}{l}9.37 \\
(0.617)\end{array}$ \\
\hline Wheat midds/millrun & 3 & $\begin{array}{l}90.58 \\
(0.466)\end{array}$ & $\begin{array}{l}94.57 \\
(0.081)\end{array}$ & $\begin{array}{l}18.48 \\
(0.361)\end{array}$ & $\begin{array}{l}2.28 \\
(0.050)\end{array}$ & $\begin{array}{l}38.50 \\
(3.000)\end{array}$ & $\begin{array}{l}37.33 \\
(1.203)\end{array}$ & $\begin{array}{l}45.90 \\
(1.900)\end{array}$ & $\begin{array}{l}11.48 \\
(0.433)\end{array}$ & $\begin{array}{l}2.78 \\
(0.165)\end{array}$ & $\begin{array}{l}24.13 \\
(1.866)\end{array}$ & $\begin{array}{l}3.22 \\
(0.387)\end{array}$ & $\begin{array}{l}2.60 \\
(0.158)\end{array}$ \\
\hline
\end{tabular}

a Dry matter.

b Organic matter.

c Crude protein.

d Acid detergent insoluble $\mathrm{CP}$, an estimate of indigestible $\mathrm{CP}(\mathrm{g} / 100 \mathrm{~g}$ of $\mathrm{CP})$.

e Soluble CP (g/100 g of CP).

f $30 \mathrm{~h}$ ruminal in vitro amylase-treated neutral detergent fiber (aNDF) digestibility ( $\mathrm{g} / 100 \mathrm{~g}$ of aNDF).

$g$ aNDF expressed exclusive of residual ash.

${ }^{h}$ Acid detergent fiber expressed exclusive of residual ash.

${ }^{\mathrm{i}}$ Lignin assayed with sulphuric acid.

j Standard deviation.

${ }^{\mathrm{k}}$ Not determined.

11 less sample for SolCP, $\mathrm{dNDF}_{30}$ and fat. 
Table 2

Chemical analysis (+standard deviation if enough samples were collected) of forages ( $\mathrm{g} / 100 \mathrm{~g} \mathrm{DM}$ ) used in the total mixed rations of the $16 \mathrm{dairies}$.

\begin{tabular}{|c|c|c|c|c|c|c|c|c|c|c|c|c|c|}
\hline & $n$ & $\mathrm{DM}^{\mathrm{a}}$ & $\mathrm{OM}^{\mathrm{b}}$ & $\mathrm{CP}^{\mathrm{c}}$ & ADICPd & SolCPe & aNDFom $^{g}$ & $\mathrm{dNDF}_{30} \mathrm{f}$ & ADFom $^{\mathrm{h}}$ & Lignin $(\mathrm{sa})^{\mathrm{i}}$ & Starch & Fat & Sugars \\
\hline Alfalfa chop ${ }^{k}$ & 7 & $\begin{array}{l}62.31 \\
(10.677)^{\mathrm{j}}\end{array}$ & $\begin{array}{l}88.25 \\
(0.456)\end{array}$ & $\begin{array}{l}21.84 \\
(0.836)\end{array}$ & $\begin{array}{l}5.51 \\
(0.412)\end{array}$ & $\begin{array}{l}40.73 \\
(1.780)\end{array}$ & $\begin{array}{l}37.12 \\
(0.910)\end{array}$ & $\begin{array}{l}40.00 \\
(1.590)\end{array}$ & $\begin{array}{l}32.35 \\
(0.690)\end{array}$ & $\begin{array}{l}6.01 \\
(0.152)\end{array}$ & $\begin{array}{l}1.56 \\
(0.095)\end{array}$ & $\begin{array}{l}1.46 \\
(0.084)\end{array}$ & $\begin{array}{l}2.92 \\
(0.465)\end{array}$ \\
\hline Alfalfa hay & 11 & $\begin{array}{l}92.25 \\
(0.264)\end{array}$ & $\begin{array}{l}89.12 \\
(0.365)\end{array}$ & $\begin{array}{l}21.06 \\
(0.764)\end{array}$ & $\begin{array}{l}5.31 \\
(0.243)\end{array}$ & $\begin{array}{l}35.81 \\
(0.670)\end{array}$ & $\begin{array}{l}35.86 \\
(1.230)\end{array}$ & $\begin{array}{l}37.82 \\
(1.400)\end{array}$ & $\begin{array}{l}29.44 \\
(1.200)\end{array}$ & $\begin{array}{l}5.48 \\
(0.324)\end{array}$ & $\begin{array}{l}1.87 \\
(0.147)\end{array}$ & $\begin{array}{l}1.52 \\
(0.096)\end{array}$ & $\begin{array}{l}4.24 \\
(0.268)\end{array}$ \\
\hline Alfalfa hay $\mathrm{HQ}^{\mathrm{l}}$ & 3 & $\begin{array}{l}92.37 \\
(0.384)\end{array}$ & $\begin{array}{l}89.79 \\
(0.632)\end{array}$ & $\begin{array}{l}21.22 \\
(1.153)\end{array}$ & $\begin{array}{l}5.74 \\
(0.903)\end{array}$ & $\begin{array}{l}36.15 \\
-\end{array}$ & $\begin{array}{l}36.53 \\
(2.230)\end{array}$ & $\begin{array}{l}31.92 \\
-\end{array}$ & $\begin{array}{l}29.37 \\
(1.157)\end{array}$ & $\begin{array}{l}5.57 \\
(0.176)\end{array}$ & $\begin{array}{l}2.17 \\
(0.367)\end{array}$ & $\begin{array}{l}1.55 \\
(0.090)\end{array}$ & $\begin{array}{l}4.10 \\
(0.404)\end{array}$ \\
\hline Alfalfa hay LQ ${ }^{1}$ & 3 & $\begin{array}{l}92.27 \\
(0.371)\end{array}$ & $\begin{array}{l}86.84 \\
(0.389)\end{array}$ & $\begin{array}{l}22.75 \\
(0.729)\end{array}$ & $\begin{array}{l}6.04 \\
(1.049)\end{array}$ & $\begin{array}{l}37.61 \\
-\end{array}$ & $\begin{array}{l}35.60 \\
(1.660)\end{array}$ & $\begin{array}{l}34.47 \\
-\end{array}$ & $\begin{array}{l}29.67 \\
(1.580)\end{array}$ & $\begin{array}{l}5.53 \\
(0.406)\end{array}$ & $\begin{array}{l}1.20 \\
(0.265)\end{array}$ & $\begin{array}{l}1.22 \\
-\end{array}$ & $\begin{array}{l}2.97 \\
(0.578)\end{array}$ \\
\hline Alfalfa silage & 4 & $\begin{array}{l}43.33 \\
(5.494)\end{array}$ & $\begin{array}{l}86.16 \\
(0.814)\end{array}$ & $\begin{array}{l}25.49 \\
(1.297)\end{array}$ & $\begin{array}{l}5.60 \\
(0.171)\end{array}$ & $\begin{array}{l}70.83 \\
(4.420)\end{array}$ & $\begin{array}{l}30.88 \\
(0.600)\end{array}$ & $\begin{array}{l}43.28 \\
(2.490)\end{array}$ & $\begin{array}{l}26.88 \\
(0.940)\end{array}$ & $\begin{array}{l}5.40 \\
(0.100)\end{array}$ & $\begin{array}{l}<0.50 \\
-\end{array}$ & $\begin{array}{l}3.01 \\
(0.430)\end{array}$ & $\begin{array}{l}0.48 \\
(0.293)\end{array}$ \\
\hline Corn earlage & 1 & 60.08 & 97.24 & 8.35 & 37.43 & 66.15 & 21.15 & 64.30 & 10.60 & 1.00 & 53.10 & 3.09 & 0.90 \\
\hline Corn silage & 15 & $\begin{array}{l}31.81 \\
(0.718)\end{array}$ & $\begin{array}{l}92.85 \\
(0.226)\end{array}$ & $\begin{array}{l}7.91 \\
(0.173)\end{array}$ & $\begin{array}{l}8.81 \\
(0.440)\end{array}$ & $\begin{array}{l}67.30 \\
(1.120)\end{array}$ & $\begin{array}{l}42.45 \\
(0.890)\end{array}$ & $\begin{array}{l}51.02 \\
(1.080)\end{array}$ & $\begin{array}{l}26.95 \\
(0.570)\end{array}$ & $\begin{array}{l}2.94 \\
(0.131)\end{array}$ & $\begin{array}{l}24.66 \\
(0.903)\end{array}$ & $\begin{array}{l}3.17 \\
(0.171)\end{array}$ & $\begin{array}{l}<0.20 \\
-\end{array}$ \\
\hline Oat straw & 1 & 92.50 & 91.70 & 8.19 & 7.63 & 34.42 & 56.50 & 57.98 & 34.10 & 3.60 & 9.20 & 1.52 & 5.60 \\
\hline Rice straw & 1 & 93.00 & 84.67 & 4.40 & 29.83 & 29.55 & 63.70 & 46.52 & 38.50 & 4.40 & 3.00 & 2.02 & 2.90 \\
\hline Wheat silage & 5 & $\begin{array}{l}33.66 \\
(2.095)\end{array}$ & $\begin{array}{l}88.14 \\
(0.892)\end{array}$ & $\begin{array}{l}10.60 \\
(0.885)\end{array}$ & $\begin{array}{l}9.67 \\
(1.106)\end{array}$ & $\begin{array}{l}74.39 \\
(1.270)\end{array}$ & $\begin{array}{l}48.22 \\
(1.901)\end{array}$ & $\begin{array}{l}51.60 \\
(2.030)\end{array}$ & $\begin{array}{l}30.84 \\
(1.225)\end{array}$ & $\begin{array}{l}4.08 \\
(0.166)\end{array}$ & $\begin{array}{l}9.98 \\
(2.303)\end{array}$ & $\begin{array}{l}2.62 \\
(0.234)\end{array}$ & $\begin{array}{l}2.37 \\
-\end{array}$ \\
\hline Wheat straw & 2 & 92.75 & 90.55 & 8.51 & 9.92 & 34.68 & 57.05 & 45.73 & 34.75 & 4.10 & 9.90 & 0.99 & 4.55 \\
\hline
\end{tabular}

a Dry matter.

b Organic matter.

c Crude protein.

d Acid detergent insoluble $\mathrm{CP}$, an estimate of indigestible $\mathrm{CP}(\mathrm{g} / 100 \mathrm{~g}$ of $\mathrm{CP})$.

e Soluble CP (g/100 g of CP).

f $30 \mathrm{~h}$ ruminal in vitro amylase-treated neutral detergent fiber (aNDF) digestibility ( $\mathrm{g} / 100 \mathrm{~g}$ of aNDF).

$\mathrm{g}$ aNDF expressed exclusive of residual ash.

${ }^{\text {h }}$ Acid detergent fiber expressed exclusive of residual ash.

${ }^{\mathrm{i}}$ Lignin assayed with sulphuric acid.

j Standard deviation.

${ }^{\mathrm{k}}$ Alfalfa chop were collected twice at 2-4-day intervals. Analyzed component values were averaged.

${ }^{1}$ High (HQ) and Low (LQ) quality alfalfa as specified by each farm. 
Table 3

Chemical analysis ( $\mathrm{g} / 100 \mathrm{~g}$ dry matter) of high group total mixed rations sampled at the 16 dairies.

\begin{tabular}{|c|c|c|c|c|c|c|c|c|c|c|c|c|c|c|c|c|c|c|}
\hline Farm number & 1 & 2 & 3 & 4 & 5 & 6 & $7^{\mathrm{a}}$ & $8^{\mathrm{a}}$ & 9 & 10 & 11 & 12 & 13 & $14^{\mathrm{a}}$ & 15 & 16 & Avg & $\mathrm{NRC}^{\mathrm{b}}$ \\
\hline Dry matter & 55.20 & 61.63 & 55.51 & 57.20 & 61.65 & 59.60 & 63.10 & 59.29 & 62.22 & 52.00 & 53.95 & 59.79 & 45.20 & 63.42 & 61.62 & 58.53 & 58.12 & \\
\hline $\mathrm{OM}^{\mathrm{c}}$ & 92.40 & 91.89 & 90.87 & 90.99 & 92.54 & 92.17 & 93.15 & 90.59 & 91.59 & 91.40 & 91.27 & 90.74 & 90.47 & 92.01 & 92.23 & 92.30 & 91.66 & \\
\hline Crude protein & 17.31 & 18.16 & 17.47 & 16.28 & 16.00 & 15.88 & 17.13 & 17.38 & 17.98 & 16.84 & 18.50 & 17.31 & 16.47 & 17.53 & 16.81 & 18.88 & 17.25 & $16.0-16.7$ \\
\hline ADICPd $^{d}$ & 5.44 & 5.88 & 5.72 & 7.29 & 6.45 & 7.30 & 6.38 & 6.30 & 4.45 & 5.57 & 7.09 & 5.76 & 7.63 & 6.61 & 8.37 & 8.40 & 6.61 & \\
\hline SolCPe & 39.42 & 38.39 & 37.31 & 37.92 & 35.70 & 39.68 & 34.82 & 40.39 & 40.92 & 39.16 & 41.87 & 34.53 & 39.50 & 34.77 & 40.42 & 36.86 & 38.23 & \\
\hline aNDFom $^{\mathrm{f}}$ & 27.05 & 31.40 & 27.70 & 28.95 & 31.90 & 28.80 & 31.25 & 29.90 & 30.65 & 29.05 & 32.25 & 26.25 & 32.63 & 30.65 & 29.85 & 31.65 & 30.00 & $25-33$ \\
\hline $\mathrm{dNDF}_{30} \mathrm{~g}$ & 47.72 & 52.40 & 44.26 & 46.49 & 44.11 & 41.18 & 53.75 & 41.27 & 46.85 & 46.87 & 45.50 & 46.54 & 48.03 & 46.74 & 43.33 & 47.25 & 46.39 & \\
\hline ADFom $^{\mathrm{h}}$ & 18.30 & 20.00 & 19.10 & 21.25 & 20.75 & 19.60 & 19.65 & 20.80 & 19.30 & 20.15 & 20.70 & 18.35 & 22.10 & 20.35 & 20.60 & 21.70 & 20.17 & $17-21$ \\
\hline $\operatorname{Lignin}(\mathrm{sa})^{\mathrm{i}}$ & 3.50 & 4.80 & 2.75 & 4.95 & 4.85 & 4.90 & 4.40 & 4.75 & 3.43 & 4.25 & 4.60 & 3.40 & 4.40 & 4.80 & 5.90 & 4.35 & 4.38 & \\
\hline Starch & 15.90 & 15.35 & 22.10 & 19.50 & 18.25 & 16.30 & 19.40 & 20.40 & 20.63 & 20.60 & 14.30 & 20.90 & 10.65 & 17.35 & 19.20 & 18.00 & 18.05 & \\
\hline Fat & 5.47 & 5.39 & 4.68 & 4.84 & 5.14 & 5.19 & 5.95 & 5.04 & 5.09 & 5.09 & 7.03 & 7.06 & 6.20 & 5.84 & 5.30 & 7.62 & 5.68 & \\
\hline Sugars & 4.90 & 3.25 & 2.95 & 3.25 & 4.55 & 4.90 & 3.55 & 3.25 & 2.03 & 1.60 & 2.65 & 1.55 & 3.18 & 3.35 & 4.15 & 2.50 & 3.23 & \\
\hline $\mathrm{NE}_{\mathrm{L}}(\mathrm{MJ} / \mathrm{kg})^{\mathrm{j}}$ & 7.50 & 7.35 & 7.07 & 7.09 & 6.98 & 7.04 & 7.60 & 6.80 & 7.11 & 7.19 & 7.10 & 7.56 & 7.03 & 7.21 & 7.05 & 7.37 & 7.19 & 6.74 \\
\hline $\mathrm{Ca}$ & 0.96 & 0.82 & 1.03 & 0.91 & 0.79 & 0.72 & 0.64 & 1.04 & 0.76 & 0.94 & 0.85 & 1.15 & 0.85 & 0.83 & 0.88 & 0.93 & 0.88 & $0.60-0.67$ \\
\hline$P$ & 0.43 & 0.53 & 0.36 & 0.42 & 0.44 & 0.48 & 0.44 & 0.50 & 0.43 & 0.42 & 0.50 & 0.50 & 0.49 & 0.46 & 0.42 & 0.46 & 0.46 & $0.36-0.38$ \\
\hline K & 1.48 & 1.55 & 1.73 & 1.74 & 1.60 & 1.84 & 1.53 & 2.06 & 1.63 & 1.77 & 1.78 & 1.39 & 1.65 & 1.64 & 1.65 & 1.55 & 1.66 & $1.06-1.07$ \\
\hline $\mathrm{Mg}$ & 0.35 & 0.29 & 0.42 & 0.33 & 0.36 & 0.30 & 0.27 & 0.33 & 0.40 & 0.37 & 0.32 & 0.41 & 0.28 & 0.26 & 0.39 & 0.29 & 0.34 & 0.20 \\
\hline $\mathrm{S}$ & 0.25 & 0.35 & 0.25 & 0.27 & 0.27 & 0.22 & 0.29 & 0.27 & 0.34 & 0.24 & 0.31 & 0.26 & 0.30 & 0.29 & 0.28 & 0.31 & 0.28 & 0.20 \\
\hline $\mathrm{Na}$ & 0.35 & 0.36 & 0.43 & 0.52 & 0.26 & 0.38 & 0.21 & 0.41 & 0.32 & 0.50 & 0.58 & 0.51 & 0.58 & 0.38 & 0.30 & 0.38 & 0.40 & 0.22 \\
\hline $\mathrm{Cl}$ & 0.57 & 0.54 & 0.65 & 0.73 & 0.59 & 0.58 & 0.48 & 0.83 & 0.64 & 0.46 & 0.60 & 0.37 & 0.77 & 0.42 & 0.41 & 0.50 & 0.57 & $0.28-0.29$ \\
\hline \multicolumn{19}{|l|}{ ppm DM } \\
\hline $\mathrm{Zn}$ & 59.50 & 63.00 & 78.50 & 103.5 & 73.50 & 43.00 & 52.50 & 103.0 & 71.50 & 67.50 & 74.50 & 110.0 & 84.50 & 57.00 & 78.00 & 72.50 & 74.50 & $52-55$ \\
\hline $\mathrm{Mn}$ & 40.50 & 46.00 & 77.50 & 69.00 & 69.00 & 32.00 & 38.50 & 95.50 & 62.75 & 58.50 & 75.50 & 73.50 & 54.75 & 55.00 & 81.00 & 58.00 & 61.69 & 13 \\
\hline $\mathrm{Fe}$ & 282.0 & 313.5 & 410.0 & 272.5 & 164.5 & 304.5 & 188.5 & 286.0 & 385.3 & 193.5 & 269.0 & 506.5 & 295.8 & 260.0 & 170.5 & 266.0 & 285.5 & $17-18$ \\
\hline $\mathrm{Cu}$ & 10.30 & 21.35 & 18.15 & 24.30 & 12.55 & 7.95 & 6.80 & 25.65 & 12.15 & 23.15 & 13.80 & 22.65 & 14.90 & 12.80 & 12.40 & 10.00 & 15.56 & 11 \\
\hline $\mathrm{Co}$ & 0.20 & 0.50 & 1.30 & 1.20 & 0.65 & 0.20 & 0.20 & 1.05 & 1.20 & 0.30 & 0.50 & 0.50 & 0.30 & 1.00 & 0.45 & 1.25 & 0.68 & 0.11 \\
\hline $\mathrm{Se}$ & 0.35 & 0.44 & 0.39 & 0.46 & 0.52 & 0.25 & 0.25 & 0.60 & 0.44 & 0.22 & 0.47 & 0.60 & 0.63 & 0.44 & 0.53 & 0.34 & 0.43 & 0.30 \\
\hline
\end{tabular}

a Values only represent one total mixed ration sample.

${ }^{\mathrm{b}}$ NRC Values for 45 to $50 \mathrm{~kg} /$ day milk production (provide recommendations for NDF and ADF, not NDFom and ADFom).

c Organic matter.

d Acid detergent insoluble crude protein (CP), an estimate of indigestible $\mathrm{CP}(\mathrm{g} / 100 \mathrm{~g}$ of $\mathrm{CP}$ ).

e Soluble CP (g/100 g of CP).

${ }^{\mathrm{f}}$ Amylase-treated neutral detergent fiber (aNDF) expressed exclusive of residual ash.

$\mathrm{g} 30 \mathrm{~h}$ ruminal in vitro amylase-treated neutral detergent fiber (aNDF) digestibility ( $\mathrm{g} / 100 \mathrm{~g}$ of aNDF).

${ }^{\mathrm{h}}$ Acid detergent fiber expressed exclusive of residual ash.

${ }^{i}$ Lignin assayed with sulphuric acid.

${ }^{\mathrm{j}}$ Net energy available for lactation, calculated from equations utilizing chemical assays and in vitro determinations as described by Robinson et al. (2004). 


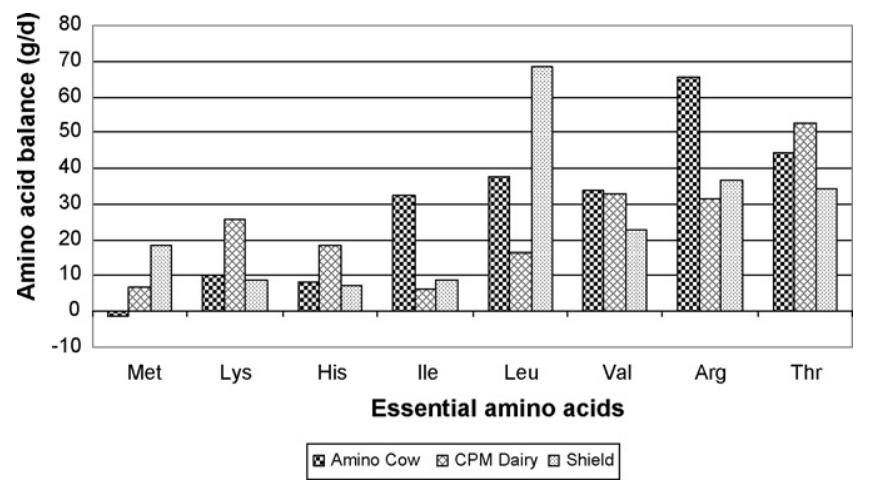

Fig. 1. Balances of average metabolizable amino acid (difference between estimated amino acid requirement and delivery) for the 16 California dairy rations as predicted by Amino Cow, CPM Dairy and Shield.

was also high consistency among the dairies in the chemical composition of the TMR and its estimated net energy for lactation.

The ingredient profiles (as $\mathrm{g} / \mathrm{kg} \mathrm{DM}$ ) of the TMR mixed for the specified pen on each of the 16 dairies (Table 4) was obtained using the on-farm computerized feed programs, which provided the actual weights of each ingredient added to the TMR during the week of the survey, or TMR mix sheets which represent the theoretical TMR. Weights were converted to $\mathrm{g} / \mathrm{kg}$ DM using the analyzed DM for each ingredient. In some cases, as in Dairy 2, accurate information on the composition of added milk cow minerals were lacking. Some ingredients were used in more than 0.8 of dairies while a few ingredients were only found on one or two. Corn products (mainly corn grain, DDG and corn silage with corn gluten feed in two and corn earlage on one of the dairies) make up $410 \mathrm{~g} / \mathrm{kg}$ of the TMR DM on average, ranging from 310 to 550 .

\subsubsection{Description of dairies}

The 16 dairies were characterized in terms of general farm management, milk production and composition, as well as intake levels and general characteristics of the cows in the specified high group (i.e., lactating cows not yet confirmed to be in calf) pen (Table 5). Milk production levels were used to assign dairy numbers starting with the lowest production of $32.8 \mathrm{~kg} / \mathrm{d}$ in Dairy 1, increasing to $51.3 \mathrm{~kg} / \mathrm{d}$ in Dairy 16 . Milk yield was dissimilar among dairies, probably because they included free stall and dry lot facilities, with 800-5000 lactating cows/dairy, milking frequencies of 2 or 3 times a day that occurred in older 'flat barns' or modern double $20-40$ parallel or herring bone milking parlours.

Average DIM were calculated together with the 10th and 90th percentiles (i.e., 10\% less than highest and 10\% higher than the lowest DIM) to exclude extreme values and provide a better representation of DIM profiles of the cows in the pens. The number of cows in the high group pen on each dairy represents only one pen, except where TMR from one load was divided between two very similar pens and uncertainties in the weight of TMR fed to each pen necessitated combination of those pens for a more accurate intake calculation.

The DM intake levels were calculated from the amount of TMR fed, estimated or calculated refusals (orts) and cow numbers, together with analyzed TMR DM values, giving average DM intakes/cow/d. All other information was obtained using the on-farm dairy herd management programs and DHIA records.

\subsection{Predicted results-model evaluation}

From model evaluations, some predictions common to the models were tabulated for each dairy (Table 6). These included predicted DM intake, estimated metabolizable protein (MP) delivery and balance (referred to in Shield as absorbable protein 'AP'), as well as the estimated delivery (g/d) and balance of metabolizable essential AA (EAA).

CPM Dairy estimated only 0.88 of measured DM intake while Amino Cow estimated 0.96 and Shield 1.02. Average estimated delivery of MP was essentially the same between CPM Dairy and Shield (2960 versus $2928 \mathrm{~g} / \mathrm{d}$ ) while Amino Cow estimated only $2594 \mathrm{~g} / \mathrm{d}$. The estimated MP balances were $1.22,1.04$ and 0.99 of requirements for Amino Cow, CPM Dairy and Shield, respectively. However there was substantial variation among dairies within model. The ratio between lysine and methionine was above 3 for Amino Cow and CPM Dairy (3.29 and 3.24) while Shield predicted only 2.61.

The summary of AA balances (Fig. 1) shows major differences among model predictions. The average balance of metabolizable methionine ranged from -1 (Amino Cow) to $18 \mathrm{~g} / \mathrm{d}$ (Shield), while lysine ranged from 9 (Shield) to $26 \mathrm{~g} / \mathrm{d}$ (CPM Dairy). The histidine balance was higher for CPM Dairy $(18 \mathrm{~g} / \mathrm{d})$ versus Amino Cow $(8 \mathrm{~g} / \mathrm{d})$ and Shield $(7 \mathrm{~g} / \mathrm{d})$. The isoleucine balance was much higher for Amino Cow (32 g/d) versus CPM Dairy (6g/d) and Shield $(9 \mathrm{~g} / \mathrm{d})$. Leucine balances varied among models from a low of $16 \mathrm{~g} / \mathrm{d}$ (CPM Dairy) to $37 \mathrm{~g} / \mathrm{d}$ (Amino Cow) and $69 \mathrm{~g} / \mathrm{d}$ (Shield). The valine balance was lower for Shield (23 g/d) 
Table 4

Ingredient profiles ( $\mathrm{g} / 100 \mathrm{~g}$ dry matter) of high group total mixed rations sampled at the 16 dairies.

\begin{tabular}{|c|c|c|c|c|c|c|c|c|c|c|c|c|c|c|c|c|}
\hline Farm number & 1 & $2^{\mathrm{a}}$ & 3 & 4 & 5 & 6 & 7 & 8 & 9 & 10 & 11 & 12 & 13 & 14 & 15 & 16 \\
\hline \multicolumn{17}{|l|}{ Forages: } \\
\hline Alfalfa chop & & & & & 9.73 & & & 24.17 & & 20.52 & 2.10 & & 3.76 & 5.72 & 20.48 & \\
\hline Alfalfa hay & 22.62 & 16.85 & 19.27 & 18.90 & 8.18 & 21.75 & & & 23.82 & 5.13 & 7.67 & 20.60 & 10.31 & 20.10 & & 14.38 \\
\hline Alfalfa silage & & 7.24 & 4.69 & & & & & & & & 3.93 & & & & & 7.76 \\
\hline Corn earlage & & & & & & & & & 16.5 & & & & & & & \\
\hline Corn silage & 16.31 & 19.56 & 23.14 & & 18.39 & 13.43 & 12.04 & 21.79 & 23.26 & 23.80 & 14.77 & 14.85 & 11.02 & 7.13 & 12.92 & 21.84 \\
\hline Oat straw/hay & & & & & & & & & 1.65 & & & 1.59 & & & & \\
\hline Rice straw & & & & & & & & & & & & & 1.85 & & & \\
\hline Wheat silage & & & & 14.81 & & & 14.65 & & & & 5.01 & & 12.43 & 8.31 & & \\
\hline Wheat straw/hay & & & & & 1.52 & & & & & & & & & & 0.50 & \\
\hline \multicolumn{17}{|c|}{ Plant products, grains and seeds } \\
\hline Almond hulls & 7.24 & 10.90 & 2.80 & 8.70 & 13.39 & 22.01 & 4.94 & 6.99 & & & 11.34 & & 3.84 & 2.56 & 15.26 & 7.85 \\
\hline Barley, rolled & & & & & & & 5.43 & & & & & & & & & \\
\hline Beet pulp shreds & & & & & & & 7.90 & & & & & & & 3.62 & & \\
\hline Brandy pomace & & & & & & & & & & & & & 1.23 & & & \\
\hline Canola pellets & & 6.10 & & 4.54 & & & 7.42 & & & & 3.78 & & 8.22 & 7.46 & 8.30 & 7.94 \\
\hline Carrot pulp & & & & 4.66 & & & & & & & & & & & & \\
\hline Citrus pulp & 3.37 & & & & & & & & & & & 9.27 & & & 3.01 & \\
\hline Corn grain, flaked & 15.02 & 10.03 & 18.43 & 24.77 & 17.64 & 15.18 & 18.69 & 15.70 & 8.40 & 19.81 & 16.68 & 26.50 & 8.49 & 17.90 & 17.87 & 15.33 \\
\hline Corn grain, ground & & & 1.64 & & & & & & & & & & & & & \\
\hline Corn gluten feed & & 6.12 & & & & & & & & & & & 3.65 & & & \\
\hline Corn gluten meal & & & 0.34 & & & & & & & & & & & & & \\
\hline Cottonseed, whole linted & 4.50 & & 8.48 & 6.52 & 6.25 & 6.62 & & 6.51 & 6.67 & 7.75 & 8.07 & 12.05 & 6.87 & & 6.20 & 9.60 \\
\hline Cottonseed, ground pima & & 6.24 & & & & & 11.39 & & & & & & & 10.06 & & \\
\hline Cottonseed, meal & & & & & & & & & & 6.27 & & & & & & \\
\hline Distillers grains, dry & 8.97 & 9.22 & 3.50 & 7.35 & 6.77 & 8.53 & 7.12 & 4.98 & 6.58 & 5.95 & 3.17 & 4.76 & 2.87 & 5.68 & & 10.30 \\
\hline Distillers grains, wet & & & & & & & & & & & 5.50 & & 6.23 & & 5.60 & \\
\hline Linseed, meal & & & & & & & & & 7.79 & & & & & & & \\
\hline Linseed, pellets & & & & & 6.76 & & & & & & & & & & & \\
\hline Raisin tailings & & & & & & & & & & & & & 2.76 & & & \\
\hline Rice bran & 2.64 & & & & & & & & & & & & & & & \\
\hline Soy hulls & & & 1.69 & & & & & & & & & & & & & \\
\hline Soybean, meal & 6.17 & & 6.63 & 5.47 & & 6.71 & & 6.70 & & & & 5.51 & & & & \\
\hline Soyplus ${ }^{\mathrm{b}}$ & & & 1.09 & & & & & 0.41 & & & & & & & & \\
\hline Wheat midds/millrun & 5.28 & & & & 8.15 & & 7.61 & 4.27 & & & 7.65 & & & 8.23 & 6.00 & \\
\hline \multicolumn{17}{|l|}{ Miscellaneous: } \\
\hline Almond shells & & & & & & & & & & & & & & & & 0.23 \\
\hline Blood meal & & & 0.72 & & & & & & & & & & & & & \\
\hline Corn/distillers syrup & & & & & & & 0.88 & & & & & & & 0.69 & & \\
\hline Fat (animal) & 0.97 & & & & & & & & & & & & 0.49 & & & \\
\hline Fat (liquid) & & & & & & & & 0.69 & & & & & & & & \\
\hline Fat (rumen inert) & & & 1.09 & 1.28 & & & 1.41 & 0.36 & 0.77 & 1.81 & & & 3.93 & 1.01 & 0.63 & 2.26 \\
\hline Fish meal & & & & & & & & 0.40 & & & & & & & & \\
\hline Generator $\mathrm{D}^{c}$ & & & 0.0004 & & & & & & & & 0.02 & & & & & \\
\hline Millrun+tallow mix & & & & & & & & & & & 3.68 & & & & & \\
\hline Mineral mixes & 0.69 & $7.73^{\mathrm{d}}$ & 2.19 & 1.51 & 1.78 & 2.10 & 0.52 & 0.35 & 0.96 & 2.19 & 1.48 & $4.44^{\mathrm{d}}$ & 2.13 & 1.53 & 1.87 & 0.58 \\
\hline Molasses & & & 0.97 & & 1.42 & & & 3.45 & 2.33 & & & & & & 1.06 & \\
\hline Prolac ${ }^{e}$ & & & & & & & & & 0.69 & & & & & & & \\
\hline Salt & & & & & & & & & & & & & & & & 0.57 \\
\hline
\end{tabular}


Table 4 (Continued)

\begin{tabular}{|c|c|c|c|c|c|c|c|c|c|c|c|c|c|c|c|c|}
\hline Farm number & 1 & $2^{\mathrm{a}}$ & 3 & 4 & 5 & 6 & 7 & 8 & 9 & 10 & 11 & 12 & 13 & 14 & 15 & 16 \\
\hline Sodium Bicarbonate & 0.49 & & 0.74 & 0.99 & & 0.73 & & 0.45 & 0.59 & 0.92 & 0.77 & & 0.90 & & & 0.57 \\
\hline Urea & 0.29 & & 0.14 & 0.46 & & 0.60 & & 0.37 & & 0.56 & 0.33 & 0.25 & 0.26 & & 0.28 & 0.39 \\
\hline Water & & & 0.02 & 0.03 & 0.01 & & & & & & & & & & & \\
\hline WCS replacer $\mathrm{f}^{\mathrm{f}}$ & & & & & & & & & & & 1.84 & & & & & \\
\hline Whey (liquid) & 5.13 & & 2.38 & & & 2.34 & & 2.31 & & 5.30 & 2.22 & & 8.76 & & & \\
\hline Yeast & 0.30 & & & & & & & & & & & 0.19 & & & & 0.40 \\
\hline $\begin{array}{l}\text { Total amount of corn } \\
\text { products used }\end{array}$ & 40.30 & 44.93 & 47.05 & 32.12 & 42.80 & 37.14 & 38.73 & 42.47 & 54.74 & 49.56 & 40.12 & 46.11 & 32.26 & 31.40 & 36.39 & 47.47 \\
\hline
\end{tabular}

${ }^{a}$ Accurate information on the composition of the milk cow mineral was not provided by the dairy.

b Heat-processed, all-natural soybean meal, deliver 60\% rumen bypass protein. SoyPLUS ${ }^{\circledR}$, West Central, Ralston, IA, USA.

c Direct fed microbial. Bio-Vet Inc., Blue Mounds, WI, USA.

d Inclusion level of top mix/premix consisting of a mineral mix and other ingredients.

e Probiotic containing the lactic acid strains L. reuteri and L. acido. Vitacel ${ }^{\circledR}$ Prolac, J. Rettenmaier \& SöhneGmbH + Co. KG, Rosenberg, Germany.

f Whole cottonseed replacer, Imperial Western Products, Inc., CA, USA. 
Table 5

Description of the 16 dairies, cows and pens designated by the dairy as one of their high group pens ${ }^{a}$.

\begin{tabular}{|c|c|c|c|c|c|c|c|c|c|c|c|c|c|c|c|c|}
\hline Farm number & 1 & 2 & 3 & 4 & 5 & 6 & 7 & 8 & 9 & 10 & 11 & 12 & 13 & 14 & 15 & 16 \\
\hline \multicolumn{17}{|l|}{ General information } \\
\hline Total lactating cows & 1000 & 1143 & 3000 & 1192 & 1809 & 2772 & 824 & 5000 & 1200 & 2648 & 2200 & 4100 & 5000 & 932 & 4400 & 1378 \\
\hline Milkings/day & 2 & 2 & 2 & 2 & 2 & 3 & 2 & 2 & 3 & 2 & 2 & 3 & 3 & 2 & 2 & 3 \\
\hline \multicolumn{17}{|l|}{ Animals } \\
\hline Cows in high group pen ${ }^{\mathrm{b}}$ & 149 & 123 & 170 & 190 & 189 & 145 & $265^{c}$ & 408 & 158 & $513^{c}$ & 191 & 264 & 364 & $223^{c}$ & $587^{c}$ & 167 \\
\hline \multicolumn{17}{|l|}{ Days in milk } \\
\hline 10th \% & 84 & 46 & 57 & 99 & 29 & 22 & 112 & 36 & 89 & 94 & 63 & 91 & 31 & 35 & 37 & 42 \\
\hline Average & 221 & 97 & 87 & 132 & 86 & 87 & 199 & 108 & 210 & 157 & 111 & 160 & 92 & 133 & 124 & 88 \\
\hline 90th \% & 345 & 141 & 119 & 170 & 129 & 139 & 291 & 170 & 321 & 236 & 164 & 226 & 156 & 218 & 218 & 134 \\
\hline \multicolumn{17}{|l|}{ Parity (\%) } \\
\hline 1 & 5 & 22 & 0 & 0 & 15 & 1 & 0 & 2 & 1 & 2 & 0 & 4 & 1 & 0 & 0 & 1 \\
\hline 2 & 62 & 53 & 49 & 30 & 58 & 52 & 39 & 11 & 59 & 47 & 44 & 46 & 48 & 16 & 0 & 37 \\
\hline$>3$ & 33 & 25 & 51 & 70 & 27 & 47 & 61 & 87 & 40 & 51 & 56 & 50 & 51 & 84 & 100 & 62 \\
\hline Parity (maximum) & 6 & 8 & 7 & 9 & 5 & 8 & 6 & 10 & 5 & 8 & 9 & 8 & 6 & 9 & 10 & 9 \\
\hline \multicolumn{17}{|l|}{ Production } \\
\hline Milk yield $(\mathrm{kg} / \mathrm{d})$ & 32.8 & 37.9 & 40.3 & 40.9 & 41.2 & 41.4 & 41.7 & 42.8 & 43.3 & 45.2 & 45.4 & 46.6 & 46.7 & 47.7 & 48.5 & 51.3 \\
\hline True prot $\%$ & 3.23 & 2.91 & 2.77 & 2.88 & 2.93 & 2.87 & 3.13 & 2.81 & 3.00 & 2.72 & 2.84 & 2.95 & 2.87 & 2.92 & 3.01 & 2.73 \\
\hline Fat $\%$ & 3.32 & 3.49 & 3.19 & 3.67 & 3.14 & 3.49 & 3.54 & 3.08 & 3.68 & 3.04 & 3.32 & 3.54 & 3.19 & 3.49 & 3.45 & 3.79 \\
\hline $\operatorname{SCC}(.000)^{d}$ & 739 & 270 & 75 & 187 & 70 & 122 & 262 & 264 & 219 & 163 & 132 & 95 & 375 & 438 & 416 & 364 \\
\hline \multicolumn{17}{|l|}{ Intakes } \\
\hline As fed $(\mathrm{kg} / \mathrm{d})$ & 43.9 & 34.7 & 48.3 & 43.5 & 46.2 & 37.8 & 45.0 & 44.9 & 40.2 & 52.5 & 48.2 & 45.8 & 53.0 & 41.1 & 48.8 & 49.9 \\
\hline DM basis $(\mathrm{kg} / \mathrm{d})$ & 24.2 & 21.4 & 26.8 & 24.9 & 28.5 & 22.5 & 28.4 & 26.6 & 25.0 & 27.3 & 26.9 & 27.4 & 24.0 & 26.0 & 30.1 & 29.2 \\
\hline Total mixed ration DM \% & 55.2 & 61.6 & 55.5 & 57.2 & 61.7 & 59.6 & 63.1 & 59.3 & 62.2 & 52.0 & 54.0 & 59.8 & 45.2 & 63.4 & 61.6 & 58.5 \\
\hline
\end{tabular}

a High group pen cows are defined as cows that are lactating but have not yet been confirmed to be in calf.

b Number of cows in the single high group pen used for the survey.

c Number of cows in two, very similar pens, fed from the same truck, combined.

d Somatic cell count. 
Table 6

Protein and amino acid status of the high group rations according to 'Amino Cow', 'CPM Dairy' and 'Shield'.

\begin{tabular}{|c|c|c|c|c|c|c|c|c|c|c|c|c|c|c|c|c|c|}
\hline Farm number & 1 & 2 & 3 & 4 & 5 & 6 & 7 & 8 & 9 & 10 & 11 & 12 & 13 & 14 & 15 & 16 & Ave \\
\hline \multicolumn{18}{|c|}{$\mathrm{DM}^{\mathrm{a}}$ intake $(\mathrm{kg} / \mathrm{d})$} \\
\hline Measured & 24.2 & 21.4 & 26.8 & 24.9 & 28.5 & 22.5 & 28.4 & 26.6 & 25.0 & 27.3 & 24.5 & 27.4 & 24.0 & 26.0 & 30.1 & 29.2 & 26.1 \\
\hline \multicolumn{18}{|l|}{ Predicted } \\
\hline Amino Cow & 22.2 & 23.8 & 23.6 & 25.0 & 23.7 & 24.4 & 25.0 & 24.4 & 25.7 & 25.0 & 25.6 & 26.4 & 25.4 & 26.6 & 26.7 & 27.9 & 25.1 \\
\hline CPM Dairy & 20.1 & 21.6 & 21.2 & 22.9 & 21.8 & 22.0 & 22.4 & 22.2 & 23.1 & 22.4 & 23.4 & 24.2 & 23.4 & 24.3 & 24.5 & 25.8 & 22.8 \\
\hline Shield & 22.7 & 25.9 & 28.5 & 30.5 & 24.9 & 25.4 & 23.1 & 25.9 & 26.7 & 27.9 & 27.4 & 24.5 & 27.2 & 28.3 & 27.0 & 28.1 & 26.5 \\
\hline \multicolumn{18}{|c|}{ MP Delivery $(\mathrm{g} / \mathrm{d})^{\mathrm{b}}$} \\
\hline Amino Cow & 2447 & 2261 & 2769 & 2516 & 2592 & 2236 & 2704 & 2740 & 2622 & 2764 & 2537 & 2560 & 2317 & 2631 & 2858 & 2948 & 2594 \\
\hline CPM Dairy & 2805 & 2389 & 3212 & 2853 & 3143 & 2527 & 3246 & 3119 & 2712 & 3059 & 2903 & 3207 & 2512 & 3045 & 3422 & 3200 & 2960 \\
\hline Shield & 2844 & 2545 & 2884 & 2566 & 3104 & 2495 & 3130 & 3026 & 2886 & 3043 & 2855 & 3160 & 2587 & 3140 & 3279 & 3299 & 2928 \\
\hline \multicolumn{18}{|l|}{ MP bal $(\mathrm{g} / \mathrm{d})^{\mathrm{c}}$} \\
\hline Amino Cow & 727 & 457 & 974 & 581 & 644 & 279 & 616 & 782 & 521 & 769 & 470 & 376 & 129 & 399 & 523 & 703 & 559 \\
\hline CPM Dairy & 433 & -7 & 608 & 195 & 343 & -135 & 277 & 393 & -165 & 221 & -9 & 228 & -419 & 21 & 122 & 20 & 133 \\
\hline Shield & 148 & -193 & 398 & 297 & 306 & -284 & -228 & 109 & -227 & 169 & -200 & -95 & -653 & -275 & 0 & 92 & -40 \\
\hline \multicolumn{18}{|l|}{$\operatorname{mMet}(\mathrm{g})^{\mathrm{d}}$} \\
\hline Amino Cow & 51 & 47 & 57 & 53 & 53 & 46 & 56 & 58 & 55 & 58 & 53 & 52 & 49 & 55 & 60 & 61 & 54 \\
\hline CPM Dairy & 57 & 47 & 62 & 57 & 65 & 51 & 65 & 64 & 54 & 57 & 58 & 62 & 51 & 59 & 69 & 60 & 59 \\
\hline Shield & 61 & 56 & 58 & 55 & 69 & 53 & 68 & 68 & 66 & 67 & 64 & 69 & 58 & 70 & 74 & 75 & 64 \\
\hline \multicolumn{18}{|c|}{ mMet $(\mathrm{g} / 100 \mathrm{~g} \mathrm{MP})^{\mathrm{e}}$} \\
\hline Amino Cow & 2.08 & 2.09 & 2.06 & 2.11 & 2.05 & 2.05 & 2.09 & 2.13 & 2.09 & 2.10 & 2.10 & 2.04 & 2.10 & 2.10 & 2.11 & 2.08 & 2.09 \\
\hline CPM Dairy & 2.05 & 1.96 & 1.92 & 1.99 & 2.07 & 2.03 & 1.99 & 2.04 & 2.00 & 1.88 & 2.01 & 1.93 & 2.03 & 1.93 & 2.01 & 1.88 & 1.98 \\
\hline Shield & 2.13 & 2.21 & 2.01 & 2.24 & 2.23 & 2.14 & 2.19 & 2.24 & 2.30 & 2.20 & 2.25 & 2.17 & 2.23 & 2.23 & 2.24 & 2.26 & 2.20 \\
\hline \multicolumn{18}{|l|}{ mLys (g) } \\
\hline Amino Cow & 171 & 153 & 200 & 177 & 168 & 155 & 180 & 197 & 177 & 191 & 170 & 172 & 158 & 181 & 197 & 195 & 178 \\
\hline CPM Dairy & 184 & 148 & 214 & 185 & 199 & 167 & 200 & 209 & 174 & 191 & 183 & 205 & 161 & 195 & 224 & 197 & 190 \\
\hline Shield & 167 & 148 & 174 & 160 & 167 & 146 & 172 & 179 & 164 & 171 & 160 & 176 & 147 & 184 & 183 & 176 & 167 \\
\hline \multicolumn{18}{|c|}{ mLys ( $\mathrm{g} / 100 \mathrm{~g} \mathrm{MP}$ ) } \\
\hline Amino Cow & 7.00 & 6.78 & 7.21 & 7.02 & 6.48 & 6.95 & 6.64 & 7.20 & 6.74 & 6.90 & 6.72 & 6.71 & 6.80 & 6.88 & 6.90 & 6.62 & 6.85 \\
\hline CPM Dairy & 6.56 & 6.20 & 6.67 & 6.48 & 6.33 & 6.61 & 6.16 & 6.68 & 6.43 & 6.25 & 6.29 & 6.38 & 6.40 & 6.42 & 6.54 & 6.16 & 6.41 \\
\hline Shield & 5.88 & 5.81 & 6.05 & 6.22 & 5.37 & 5.86 & 5.50 & 5.92 & 5.68 & 5.63 & 5.59 & 5.57 & 5.66 & 5.87 & 5.57 & 5.35 & 5.72 \\
\hline \multicolumn{18}{|l|}{ Lys:Met } \\
\hline Amino Cow & 3.35 & 3.26 & 3.51 & 3.34 & 3.17 & 3.37 & 3.21 & 3.40 & 3.22 & 3.29 & 3.21 & 3.31 & 3.22 & 3.29 & 3.28 & 3.20 & 3.29 \\
\hline CPM Dairy & 3.20 & 3.16 & 3.47 & 3.26 & 3.06 & 3.26 & 3.09 & 3.27 & 3.21 & 3.33 & 3.13 & 3.30 & 3.15 & 3.33 & 3.26 & 3.28 & 3.24 \\
\hline Shield & 2.76 & 2.63 & 3.01 & 2.90 & 2.41 & 2.74 & 2.51 & 2.64 & 2.48 & 2.56 & 2.49 & 2.57 & 2.54 & 2.63 & 2.49 & 2.36 & 2.61 \\
\hline \multicolumn{18}{|l|}{ mHis (g/d) } \\
\hline Amino Cow & 61 & 56 & 73 & 63 & 63 & 55 & 68 & 68 & 64 & 69 & 63 & 64 & 57 & 66 & 71 & 73 & 65 \\
\hline CPM Dairy & 72 & 61 & 90 & 75 & 80 & 65 & 87 & 81 & 70 & 80 & 76 & 82 & 67 & 83 & 93 & 85 & 78 \\
\hline Shield & 57 & 50 & 67 & 52 & 59 & 49 & 64 & 61 & 54 & 64 & 56 & 63 & 51 & 62 & 66 & 66 & 59 \\
\hline \multicolumn{18}{|l|}{ mIle (g/d) } \\
\hline Amino Cow & 137 & 124 & 153 & 141 & 138 & 124 & 143 & 156 & 144 & 153 & 138 & 138 & 128 & 143 & 157 & 158 & 142 \\
\hline CPM Dairy & 142 & 120 & 158 & 143 & 161 & 129 & 156 & 159 & 142 & 154 & 145 & 161 & 128 & 155 & 175 & 158 & 149 \\
\hline Shield & 117 & 104 & 112 & 111 & 116 & 102 & 123 & 121 & 112 & 121 & 114 & 123 & 108 & 133 & 132 & 130 & 117 \\
\hline \multicolumn{18}{|l|}{ mLeu (g/d) } \\
\hline Amino Cow & 227 & 207 & 262 & 233 & 230 & 205 & 240 & 252 & 240 & 253 & 232 & 232 & 215 & 235 & 260 & 268 & 237 \\
\hline CPM Dairy & 224 & 192 & 269 & 233 & 246 & 203 & 252 & 250 & 214 & 242 & 230 & 257 & 206 & 241 & 271 & 256 & 237 \\
\hline Shield & 248 & 216 & 256 & 214 & 260 & 216 & 267 & 259 & 232 & 259 & 251 & 275 & 232 & 269 & 284 & 286 & 252 \\
\hline \multicolumn{18}{|l|}{ mVal (g/d) } \\
\hline Amino Cow & 154 & 141 & 177 & 157 & 158 & 139 & 165 & 173 & 165 & 173 & 157 & 156 & 146 & 164 & 178 & 181 & 162 \\
\hline CPM Dairy & 160 & 138 & 189 & 164 & 183 & 146 & 184 & 179 & 163 & 178 & 168 & 180 & 148 & 180 & 201 & 188 & 172 \\
\hline Shield & 142 & 133 & 143 & 133 & 148 & 124 & 160 & 149 & 139 & 154 & 147 & 155 & 132 & 164 & 167 & 165 & 147 \\
\hline
\end{tabular}


Table 6 (Continued)

\begin{tabular}{|c|c|c|c|c|c|c|c|c|c|c|c|c|c|c|c|c|c|}
\hline Farm number & 1 & 2 & 3 & 4 & 5 & 6 & 7 & 8 & 9 & 10 & 11 & 12 & 13 & 14 & 15 & 16 & Ave \\
\hline \multicolumn{18}{|l|}{$\mathrm{mArg}(\mathrm{g} / \mathrm{d})$} \\
\hline Amino Cow & 134 & 119 & 156 & 139 & 144 & 122 & 151 & 154 & 148 & 162 & 139 & 146 & 124 & 148 & 155 & 157 & 144 \\
\hline CPM Dairy & 181 & 146 & 207 & 181 & 206 & 164 & 207 & 204 & 184 & 204 & 183 & 207 & 158 & 198 & 218 & 200 & 191 \\
\hline Shield & 141 & 126 & 148 & 131 & 149 & 120 & 151 & 154 & 142 & 168 & 137 & 154 & 122 & 157 & 160 & 152 & 145 \\
\hline \multicolumn{18}{|l|}{$\operatorname{mThr}(\mathrm{g} / \mathrm{d})$} \\
\hline Amino Cow & 134 & 123 & 152 & 138 & 137 & 122 & 143 & 152 & 142 & 151 & 137 & 136 & 126 & 143 & 156 & 157 & 141 \\
\hline CPM Dairy & 138 & 113 & 155 & 139 & 151 & 125 & 151 & 154 & 132 & 145 & 138 & 154 & 121 & 147 & 167 & 151 & 143 \\
\hline Shield & 135 & 121 & 131 & 127 & 140 & 118 & 143 & 141 & 136 & 138 & 134 & 144 & 123 & 152 & 154 & 152 & 137 \\
\hline
\end{tabular}

a Dry matter.

Metabolizable protein.

c MP balance, the difference between estimated MP requirement and delivery.

${ }^{d}$ Estimated delivery $(\mathrm{g} / \mathrm{d})$ of amino acids to the small intestine.

e Metabolizable amino acids expressed as a percentage of metabolizable crude protein. 


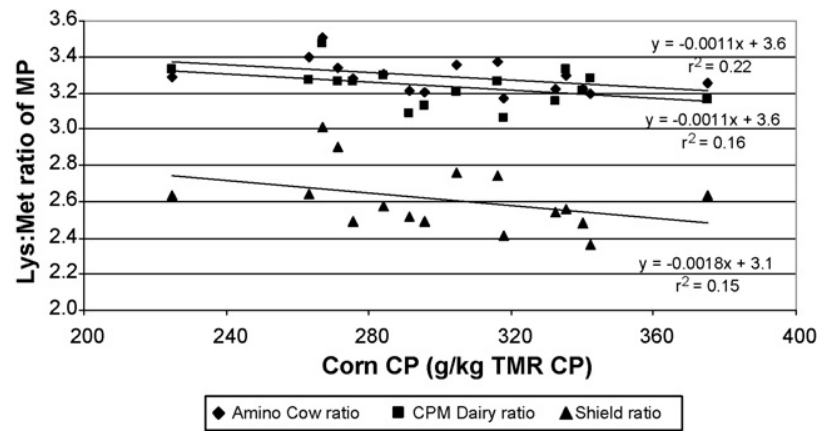

Fig. 2. The effect of corn crude protein in the total mixed ration on the ratio of Lys to Met in metabolizable protein predicted by Amino Cow, CPM Dairy and Shield.

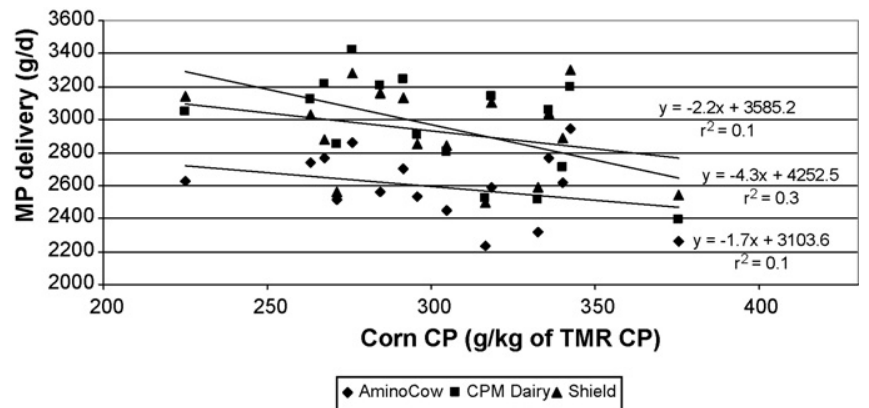

Fig. 3. The effect of corn crude protein in the total mixed ration on metabolizable protein delivery to the intestine as predicted by Amino Cow, CPM Dairy and Shield.

versus Amino Cow (33 g/d) and CPM Dairy (32 g/d). The arginine balance for Amino Cow was $65 \mathrm{~g} / \mathrm{d}$, which was much higher than the 38 and $31 \mathrm{~g} / \mathrm{d}$ for Shield and CPM Dairy. Threonine balances differed among models with CPM Dairy, Amino Cow and Shield predicting 52, 44 and $34 \mathrm{~g} / \mathrm{d}$.

\section{Discussion}

\subsection{Effect of increased contribution of corn crude protein to total TMR CP on milk production}

Even though most of the rations had a CP level slightly higher than NRC requirements (Table 3) with 200-400 g/kg of total TMR CP coming from corn products, there was no negative effect of increased levels of corn products per se on milk production, protein or fat content, suggesting that even though corn proteins made up a large proportion of total $\mathrm{CP}$ consumed, the unbalanced AA profile was either offset by inclusion of other, possibly complementary, CP sources such as canola meal, whole cottonseed, soybean meal and small amounts of animal protein sources (i.e., blood meal and fish meal) or because CP levels of some rations were relatively high (up to $188 \mathrm{~g} / \mathrm{kg} \mathrm{DM}$ ).

\subsection{Model evaluation}

Metabolic models were used to provide estimates of AA requirements and availability as there are no other accepted and published AA evaluation models providing adequate information on this set of feed ingredients that could be used to evaluate these performance results in quantitative terms.

\subsubsection{Effect of increased contribution of corn CP to total TMR CP on amino acid profile of metabolizable protein}

Even though the models did not agree on the AA profiles of protein reaching the intestine, their predictions regarding the effect of increased corn levels in the diet on these AA profiles were very consistent. As might be expected due to the low level of lysine in corn proteins, all models predicted that the lysine to methionine ratio in MP decreased as more corn protein was added to the TMR (Fig. 2), even though the ratio itself differed sharply among models. 

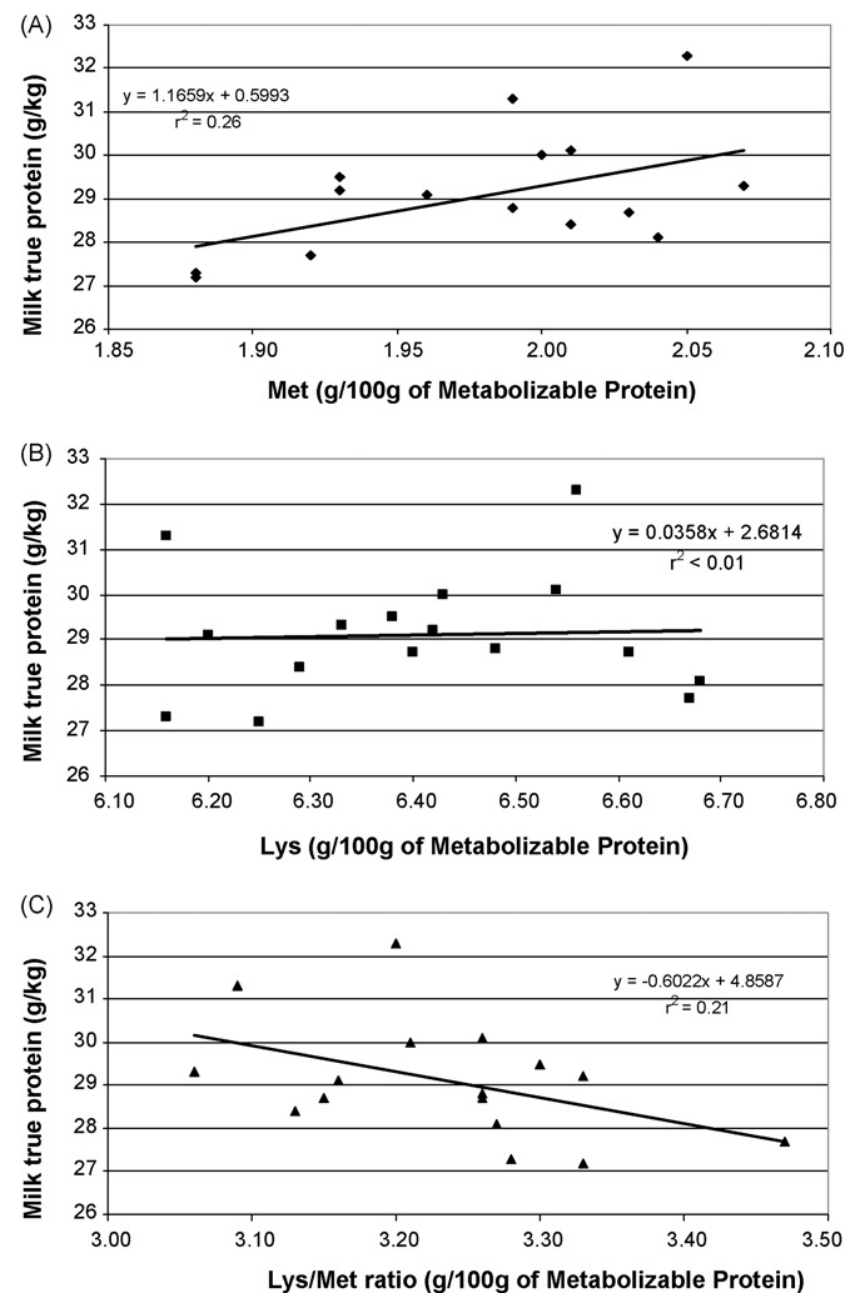

Fig. 4. The response of milk true protein proportion $(\mathrm{g} / \mathrm{kg})$ to changes in proportions of (A) Met, (B) Lys and (C) the Lys to Met ratio in metabolizable protein as predicted by CPM Dairy.

The models also suggested a decrease in MP delivery with increased contribution of corn CP to total TMR CP (Fig. 3) but none predicted any change in the proportion of methionine or lysine in MP when corn CP in the TMR increased (Table 6). Most corn proteins are higher in rumen degradable CP $(\sim 550 \mathrm{~g} / \mathrm{kg} \mathrm{CP})$ than rumen undegradable $\mathrm{CP}$, which could explain the predicted decrease of MP delivery when corn CP levels in the ration increased. That the AA levels in MP did not change, however, could be due to the increased proportional contribution of MCP (high in lysine) to total MP, delivering a much better balance of AA to the intestine. Increased MCP production could be due to better energy and N synchronization in high corn rations, therefore increasing the efficiency of microbial growth.

\subsubsection{Effect of increased contribution of corn CP to total TMR CP on milk composition}

Corn CP levels in the TMR seemed to cause a change in the predicted ratio of lysine to methionine reaching the intestine (Fig. 2) which, in turn, might have impacted milk composition. However neither the proportion, nor the ratio, of lysine and methionine in MP had any affect on either milk fat or milk true protein proportion. CPM Dairy was the only model that predicted, albeit to a very small extent $\left(r^{2}=0.21\right)$, an increase in milk protein level with a decrease in lysine to methionine ratio (Fig. 4), but this was due to increased delivery of methionine $\left(r^{2}=0.26\right)$, not a decrease in lysine $\left(r^{2}<0.01\right)$, suggesting that the decline in lysine delivery to the intestine due to high inclusion levels of corn products was not large enough to have impacted milk protein, but that the higher methionine content of corn products increased methionine delivery, with a resulting increase in milk protein proportion.

The predicted increase in methionine contribution to MP (i.e., $18.8-20.7 \mathrm{~g} / \mathrm{kg}$; Table 6 ) only yielded a small increase in milk true protein. CPM Dairy was the only model to predict a correlation between AA and milk components (i.e., milk true protein), and Shield was the only one to predict a correlation between AA and milk yield (Fig. 5). Contrary to expectations with decreased lysine (NRC, 2001), Shield predicted milk yield to increase when the ratio of lysine to methionine decreased, due to higher methionine and lower lysine proportions in MP (Fig. 6), which corresponds with AA levels in corn products. 

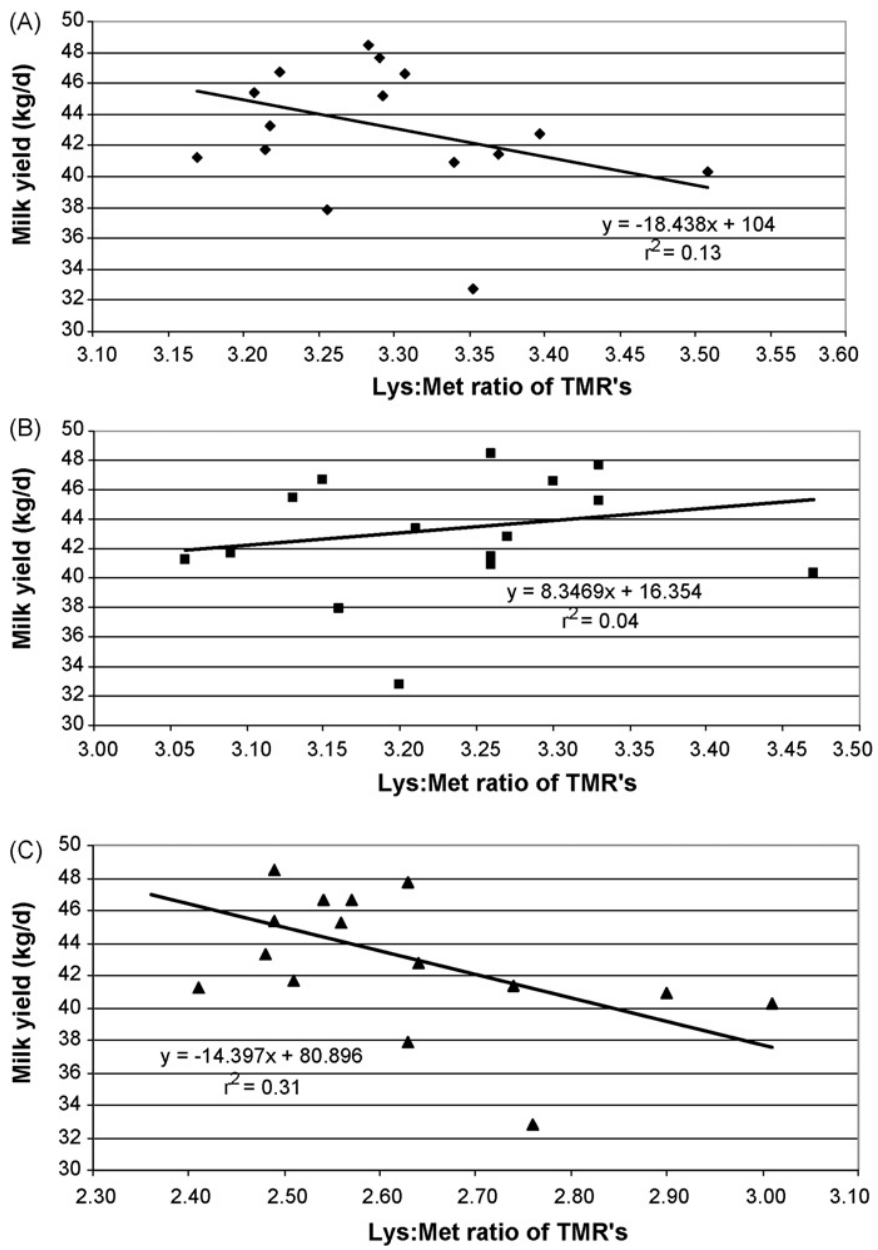

Fig. 5. The response of milk yield (kg/d) to changes in the Lys to Met ratio as predicted by (A) Amino Cow, (B) CPM Dairy and (C) Shield.

The yield increase of $18.5 \mathrm{~kg} / \mathrm{d}$, due to changes in the ratio of lysine to methionine (from 3.01 to 2.36) predicted by Shield, is much more substantive than the protein increase predicted by CPM Dairy. The possibility of increased dietary corn levels impacting milk yield therefore seems higher than for milk components.

Similar comparisons were made between other EAA and milk production (not shown), but no relationship was predicted by Amino Cow, CPM Dairy or Shield.

\subsubsection{Predicted amino acid packages}

The sequence of AA limitation (Table 7) among dairies was the same within Amino Cow (i.e., methionine, lysine, histidine, leucine, valine, isoleucine) and very similar within Shield (i.e., lysine, isoleucine, histidine, valine, arginine). In contrast, the sequence varied somewhat within CPM Dairy, although isoleucine and leucine were always (with one exception) either first or second limiting, methionine and lysine were always third or fourth limiting followed by arginine, valine and histidine.

Based upon the evaluation of each ration by each model, average AA supplementation packages were calculated to bring model estimated AA deliveries to a minimum of 1.10, 1.20 and 1.30 of model estimated requirements (Table 8). Due to differences among models in predicted AA limitation sequences, the calculated AA supplementation packages varied sharply by model. In general, Amino Cow emphasized methionine and lysine as being most limiting. CPM Dairy emphasized isoleucine and leucine, whereas Shield emphasized lysine and isoleucine. Only threonine appeared in no AA package, although arginine only appeared in CPM Dairy, and at low levels. Except at 1.10, where the sizes of the AA packages were low (i.e., 9-14 g/cow/d), CPM Dairy required AA package sizes that were 1.5 (at 1.20) to 0.7 (at 1.30) the size of those of Amino Cow and Shield (Table 8). This reflects the higher predicted animal requirements (g/d) for AA according to CPM Dairy.

Variation in predicted AA limitation sequences among models are likely due to differences in assigned AA levels of feed and MCP, and AA 'transfer coefficients' on which each model based prediction for efficiency of AA digestion, absorption and utilization. Likewise, predicted AA supply to the intestinal absorptive site depends on the default chemical composition of 

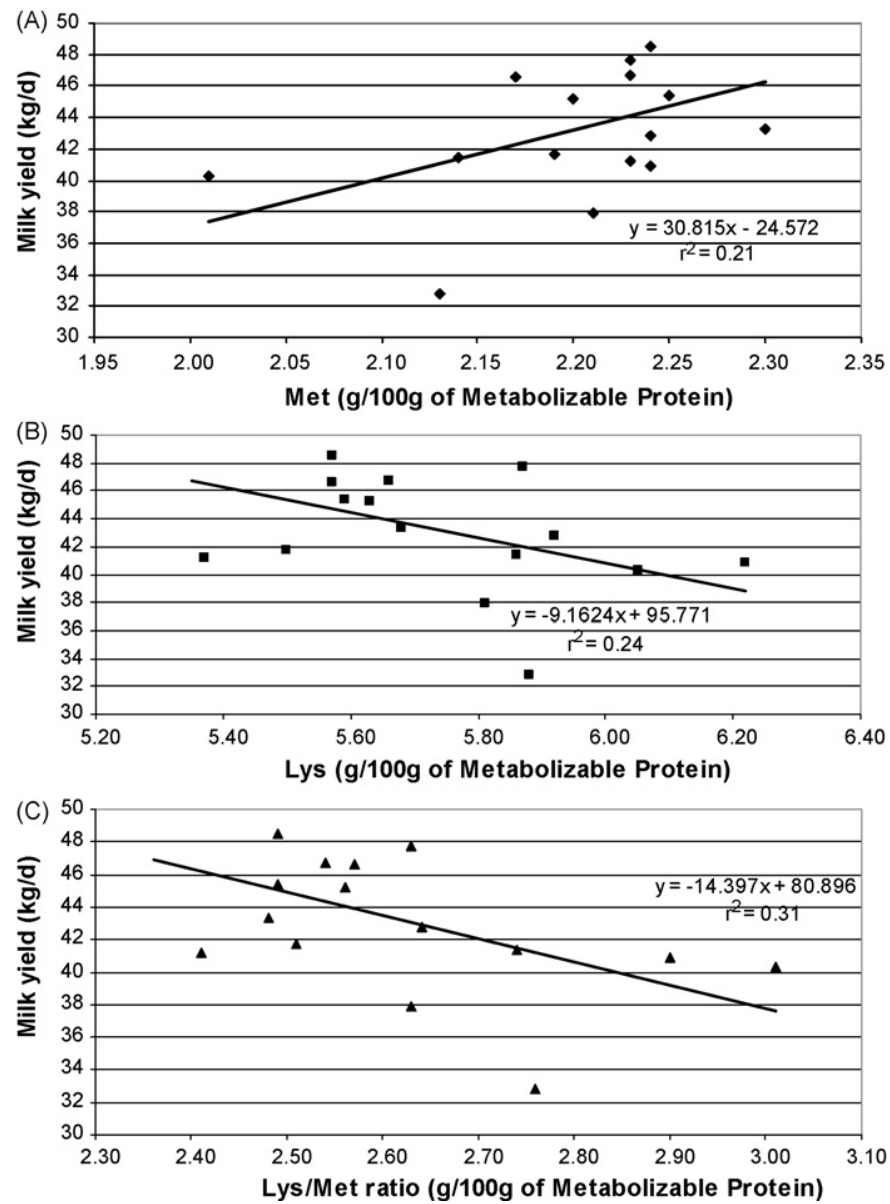

Fig. 6. The response of milk yield ( $\mathrm{kg} / \mathrm{d})$ to changes in proportions of (A) Met, (B) Lys and (C) the Lys to Met ratio in metabolizable protein predicted by Shield.

Table 7

The sequence of amino acid limitation according to 'Amino Cow', 'CPM Dairy' and 'Shield'a .

\begin{tabular}{|c|c|c|c|c|c|c|c|c|c|c|c|c|c|c|c|c|c|}
\hline Farm number & $\mathrm{Seq}^{\mathrm{b}}$ & 1 & 2 & 3 & 4 & 5 & 6 & 7 & 8 & 9 & 10 & 11 & 12 & 13 & 14 & 15 & 16 \\
\hline Amino Cow & $\begin{array}{l}1 \\
2 \\
3 \\
4\end{array}$ & Met & $\begin{array}{l}\text { Met } \\
\text { Lys } \\
\text { His } \\
\text { Leu }\end{array}$ & Met & $\begin{array}{l}\text { Met } \\
\text { Lys } \\
\text { His }\end{array}$ & $\begin{array}{l}\text { Met } \\
\text { Lys } \\
\text { His }\end{array}$ & $\begin{array}{l}\text { Met } \\
\text { Lys } \\
\text { His } \\
\text { Leu } \\
\text { Val } \\
\text { Ile }\end{array}$ & $\begin{array}{l}\text { Met } \\
\text { Lys } \\
\text { His } \\
\text { Leu }\end{array}$ & Met & $\begin{array}{l}\text { Met } \\
\text { Lys } \\
\text { His } \\
\text { Leu }\end{array}$ & $\begin{array}{l}\text { Met } \\
\text { Lys }\end{array}$ & $\begin{array}{l}\text { Met } \\
\text { Lys } \\
\text { His } \\
\text { Leu }\end{array}$ & $\begin{array}{l}\text { Met } \\
\text { Lys } \\
\text { His } \\
\text { Leu } \\
\text { Val } \\
\text { Ile }\end{array}$ & $\begin{array}{l}\text { Met } \\
\text { Lys } \\
\text { His } \\
\text { Leu } \\
\text { Val } \\
\text { Ile }\end{array}$ & $\begin{array}{l}\text { Met } \\
\text { Lys } \\
\text { His } \\
\text { Leu } \\
\text { Val } \\
\text { Ile }\end{array}$ & $\begin{array}{l}\text { Met } \\
\text { Lys } \\
\text { His } \\
\text { Leu }\end{array}$ & $\begin{array}{l}\text { Met } \\
\text { Lys } \\
\text { His }\end{array}$ \\
\hline CPM Dairy & $\begin{array}{l}2 \\
3 \\
4 \\
5 \\
6 \\
7\end{array}$ & Ile & $\begin{array}{l}\text { Ile } \\
\text { Leu } \\
\text { Lys } \\
\text { Met } \\
\text { Arg } \\
\text { Val }\end{array}$ & & $\begin{array}{l}\text { Ile } \\
\text { Leu } \\
\text { Met }\end{array}$ & $\begin{array}{l}\text { Leu } \\
\text { Ile }\end{array}$ & $\begin{array}{l}\text { Ile } \\
\text { Leu } \\
\text { Met } \\
\text { Lys } \\
\text { Arg } \\
\text { Val } \\
\text { His }\end{array}$ & $\begin{array}{l}\text { Ile } \\
\text { Leu } \\
\text { Lys } \\
\text { Met }\end{array}$ & $\begin{array}{l}\text { Ile } \\
\text { Leu }\end{array}$ & $\begin{array}{l}\text { Leu } \\
\text { Ile } \\
\text { Met } \\
\text { Lys } \\
\text { Arg } \\
\text { Val } \\
\text { His }\end{array}$ & $\begin{array}{l}\text { Ile } \\
\text { Leu } \\
\text { Met } \\
\text { Lys }\end{array}$ & $\begin{array}{l}\text { Ile } \\
\text { Leu } \\
\text { Lys } \\
\text { Met } \\
\text { Arg } \\
\text { Val }\end{array}$ & $\begin{array}{l}\text { Ile } \\
\text { Leu } \\
\text { Met } \\
\text { Lys }\end{array}$ & $\begin{array}{l}\text { Ile } \\
\text { Leu } \\
\text { Lys } \\
\text { Met } \\
\text { Arg } \\
\text { Val } \\
\text { His }\end{array}$ & $\begin{array}{l}\text { Ile } \\
\text { Leu } \\
\text { Met } \\
\text { Lys } \\
\text { Arg }\end{array}$ & $\begin{array}{l}\text { Leu } \\
\text { Ile } \\
\text { Met } \\
\text { Lys } \\
\text { Arg }\end{array}$ & $\begin{array}{l}\text { Ile } \\
\text { Met } \\
\text { Leu } \\
\text { Lys } \\
\text { Arg }\end{array}$ \\
\hline Shield & $\begin{array}{l}1 \\
2 \\
3 \\
4 \\
5 \\
6\end{array}$ & $\begin{array}{l}\text { Lys } \\
\text { His }\end{array}$ & $\begin{array}{l}\text { Lys } \\
\text { Ile } \\
\text { His }\end{array}$ & Ile & & $\begin{array}{l}\text { Lys } \\
\text { Ile } \\
\text { His }\end{array}$ & $\begin{array}{l}\text { Lys } \\
\text { Ile } \\
\text { His } \\
\text { Val }\end{array}$ & $\begin{array}{l}\text { Lys } \\
\text { Ile } \\
\text { His } \\
\text { Val } \\
\text { Arg }\end{array}$ & $\begin{array}{l}\text { Lys } \\
\text { Ile } \\
\text { His }\end{array}$ & $\begin{array}{l}\text { Lys } \\
\text { Ile } \\
\text { His } \\
\text { Val }\end{array}$ & $\begin{array}{l}\text { Lys } \\
\text { Ile }\end{array}$ & $\begin{array}{l}\text { Lys } \\
\text { Ile } \\
\text { His } \\
\text { Val }\end{array}$ & $\begin{array}{l}\text { Lys } \\
\text { Ile } \\
\text { His } \\
\text { Val }\end{array}$ & $\begin{array}{l}\text { Lys } \\
\text { Ile } \\
\text { His } \\
\text { Val } \\
\text { Arg } \\
\text { Thr }\end{array}$ & $\begin{array}{l}\text { Lys } \\
\text { Ile } \\
\text { His } \\
\text { Val }\end{array}$ & $\begin{array}{l}\text { Lys } \\
\text { Ile } \\
\text { His } \\
\text { Val }\end{array}$ & $\begin{array}{l}\text { Lys } \\
\text { Ile } \\
\text { His }\end{array}$ \\
\hline
\end{tabular}

a Only amino acids predicted to be supplied below 1.20 of requirements are listed.

b The sequence of limiting amino acids as predicted by each of the models. 
Table 8

Amino acid supplementation package sizes, and amino acid profiles (g/kg of the package), as predicted by 'Amino Cow', 'CPM Dairy' and 'Shield' to bring all amino acids to $1.30,1.20$ or 1.10 of estimated requirements.

\begin{tabular}{|c|c|c|c|c|c|c|c|c|c|}
\hline & Package g/d & Met & Lys & His & Ile & Leu & Val & Arg & Thr \\
\hline \multicolumn{10}{|l|}{ То 1.30} \\
\hline Amino Cow & 86.4 & 205 & 462 & 10 & 7 & 259 & 56 & 0 & 0 \\
\hline CPM Dairy & 143.6 & 61 & 164 & 0 & 256 & 347 & 60 & 112 & 0 \\
\hline Shield & 85.2 & 0 & 458 & 95 & 279 & 0 & 168 & 0 & 0 \\
\hline \multicolumn{10}{|l|}{ То 1.20} \\
\hline Amino Cow & 38.2 & 320 & 607 & 8 & 0 & 64 & 0 & 0 & 0 \\
\hline CPM Dairy & 61.1 & 59 & 117 & 0 & 368 & 454 & 0 & 2 & 0 \\
\hline Shield & 40.9 & 0 & 566 & 71 & 316 & 0 & 47 & 0 & 0 \\
\hline \multicolumn{10}{|l|}{ То 1.10} \\
\hline Amino Cow & 13.2 & 508 & 492 & 0 & 0 & 0 & 0 & 0 & 0 \\
\hline CPM Dairy & 13.9 & 0 & 0 & 0 & 588 & 412 & 0 & 0 & 0 \\
\hline Shield & 9.3 & 0 & 781 & 0 & 219 & 0 & 0 & 0 & 0 \\
\hline
\end{tabular}

feed components in the model ingredient libraries used to create the rations, as well as the assumed AA profiles of feed proteins escaping the rumen. The accuracy of these estimates is unknown.

\section{Conclusions}

The predicted lysine to methionine ratio in intestinally delivered protein decreased as more corn $\mathrm{CP}$ was included in the TMR, however it did not have a major impact on the final predicted AA profile of MP. Regardless of the effect that corn CP had on AA entering the intestine, the changed AA ratios in MP did not have an impact on milk component levels, and only Shield predicted an effect thereof on milk yield.

The metabolic models suggested three dramatically different AA packages with 'Amino Cow' suggesting inclusion of methionine and lysine, 'CPM Dairy' suggesting isoleucine and leucine and 'Shield' suggesting inclusion of lysine and isoleucine as first limiting AA in order to meet predicted requirements. There appears to be a high degree of consistency within model in predicting the limiting AA sequence among dairies, even though there is a substantial variation in predicted AA and MP levels delivered by the rations among diaries.

While there is sufficient consistency in the AA profiles of MP among rations to support production of a ruminally protected AA complex, which could balance model predicted AA profile, thereby leading to increased animal productivity and efficiency of utilization of nutrients, there is no absolute way to decide which model is most accurate. However, since Shield evaluations suggested a higher correlation between AA (both Lys and Met) and milk production, and predicted AA ratios with milk responses related to these ratios, using the ruminally protected AA package predicted by Shield is supported.

\section{Acknowledgements}

This survey was conducted on commercial dairies and the authors thank all of the farmers, farm advisors and nutritionists for their cooperation. Funding was provided by the University of California, Davis, USA.

\section{References}

Amino Cow, 2007. The Mepron Dairy Ration Evaluator. Version 3.5.1. Degussa Corp., Hanau, Germany.

Belyea, R.L., Steevens, B.J., Restrepo, R.J., Clubb, A.P., 1989. Variation in composition of by-product feeds. J. Dairy Sci. 72, $2339-2345$.

Burris, W.R., Boling, J.A., Bradley, N.W., Young, A.W., 1976. Abomasal lysine infusion in steers fed a urea supplemented diet. J. Anim. Sci. 42, 699-705.

CPM Dairy, 2006. Version 3.0. Department of Animal Sciences, Cornell University/Univ. Pennsylvania/W.H. Miner Agricultural Research Institute, Ithaca, $\mathrm{NY} /$ Kennett Square, PA/Chazy, NY, USA.

Derrig, R.G., Clark, J.H., Davis, C.L., 1974. Effect of abomasal infusion of sodium caseinate on milk yield, nitrogen utilization and amino acid nutrition of the dairy cow. J. Nutr. 104, 151-159.

Liu, C., Schingoethe, D.J., Stegeman, G.A., 2000. Corn distillers grains versus a blend of protein supplements with or without ruminally protected amino acids for lactating cows. J. Dairy Sci. 83, 2075-2084.

National Research Council, 2001. Nutrient Requirements of Dairy Cattle, 7th revised ed. National Academy of Science Press, Washington, DC, USA.

Nichols, J.R., Schingoethe, D.J., Maiga, H.A., Brouk, M.J., Piepenbrink, M.S., 1998. Evaluation of corn distillers grains and ruminally protected lysine and methionine for lactating dairy cows. J. Dairy Sci. 81, 482-491.

Piepenbrink, M.S., Schingoethe, D.J., 1998. Ruminal degradation, amino acid composition, and estimated intestinal digestibility of four protein supplements. J. Dairy Sci. 81, 454-461.

Robinson, P.H., 2010. Impacts of manipulating ration metabolizable lysine and methionine levels on the performance of lactating dairy cows: a systematic review of the literature. Livest. Sci. 127, 115-126.

Robinson, P.H., 2009. SHIELD Dairy Ration Evaluator. Department of Animal Science, UC Davis, Davis, CA, USA

Robinson, P.H., Givens, D.I., Getachew, G., 2004. Evaluation of NRC, UC Davis and ADAS approaches to estimate the metabolizable energy values of feeds at maintenance energy intake from equations utilizing chemical assays and in vitro determinations. Anim. Feed Sci. Technol. 114, 75-90.

Schwab, C.G., Muise, S.J., Hylton, W.E., Moore, J.J., 1982. Response to abomasal infusion of methionine of weaned dairy calves fed a complete pelleted starter ration based on by-product feeds. J. Dairy Sci. 65, 1950-1961.

Schwab, C.G., Satter, L.D., Clay, A.B., 1976. Response of lactating dairy cows to abomasal infusion of amino acids. J. Dairy Sci. 59, 1254-1270.

St-Pierre, N.R., Thraen, C.S., 1999. Animal grouping strategies, sources of variation, and economic factors affecting nutrient balance on dairy farms. J. Anim. Sci. $77,72-83$. 
Swanepoel, N., Robinson, P.H., Erasmus, L.J., 2010. Amino acid needs of lactating dairy cows: impact of feeding lysine in a ruminally protected form on productivity of lactating dairy cows. Anim. Feed Sci. Technol. 157, 79-94.

Vanhatalo, A., Huhtanen, P., Toivonen, V., Varvikko, T., 1999. Response of dairy cows fed grass silage diets to abomasal infusions of histidine alone or in combination with methionine and lysine. J. Dairy Sci. 82, 2674-2685.

Vik-Mo, L., Emery, R.S., Huber, J.T., 1974. Milk protein production in cows abomasally infused with casein or glucose. J. Dairy Sci. 57, 869-877. 